PREPARED For SUBMisSion to JCAP

\title{
Extragalactic diffuse $\gamma$-rays from dark matter annihilation: revised prediction and full modelling uncertainties
}

\author{
M. Hütten, ${ }^{a, b, c}$ C. Combet, ${ }^{c}$ D. Maurin. ${ }^{c}$ \\ ${ }^{a}$ Humboldt-Universität zu Berlin, Newtonstraße 15, D-12489 Berlin, Germany \\ ${ }^{b}$ DESY, Platanenallee 6, D-15738 Zeuthen, Germany \\ ${ }^{c}$ LPSC, Université Grenoble-Alpes, CNRS/IN2P3, 53 avenue des Martyrs, 38026 Grenoble, \\ France \\ E-mail: moritz.huetten@hu-berlin.de, celine.combet@lpsc.in2p3.fr, \\ dmaurin@lpsc.in2p3.fr
}

\begin{abstract}
Recent high-energy data from Fermi-LAT on the diffuse $\gamma$-ray background have been used to set among the best constraints on annihilating $\mathrm{TeV}$ cold dark matter candidates. In order to assess the robustness of these limits, we revisit and update the calculation of the isotropic extragalactic $\gamma$-ray intensity from dark matter annihilation. The emission from halos with masses $\geq 10^{10} \mathrm{M}_{\odot}$ provides a robust lower bound on the predicted intensity. The intensity including smaller halos whose properties are extrapolated from their higher mass counterparts is typically 5 times higher, and boost from subhalos yields an additional factor $\sim 1.5$. We also rank the uncertainties from all ingredients and provide a detailed error budget for them. Overall, our fiducial intensity is a factor 5 lower than the one derived by the FermiLAT collaboration in their latest analysis. This indicates that the limits set on extragalactic dark matter annihilations could be relaxed by the same factor. We also calculate the expected intensity for self-interacting dark matter in massive halos and find the emission reduced by a factor 3 compared to the collisionless counterpart. The next release of the CLUMPY code will provide all the tools necessary to reproduce and ease future improvements of this prediction.
\end{abstract}

Keywords: cosmic web, dark matter simulations, gamma ray experiments, semi-analytic modelling 


\section{Contents}

1 Introduction $\quad 1$

2 Modelling the $\gamma$-ray emission from extragalactic DM halos 3

3 Exotic $\gamma$-ray intensity in the collisionless CDM paradigm 5

3.1 Contribution $I_{0}$ from high-mass halos $\left(M \geq 10^{10} \mathrm{M}_{\odot}\right)$ : a robust lower bound 5

3.2 Including low-mass halos and subhalos $\quad 12$

$\begin{array}{lll}3.2 .1 & \text { Extrapolation of the mass function } & 12\end{array}$

$\begin{array}{lll}3.2 .2 & \text { Boost from DM halo substructures } & 15\end{array}$

4 Discussion $\quad 16$

$\begin{array}{lll}4.1 & \text { Comparison to other works } & 18\end{array}$

4.2 A word on IDM (SIDM $/ \gamma \mathrm{CDM})$ models 18

5 Conclusions $\quad 21$

$\begin{array}{ll}\text { A Mass conversion between different } \Delta \text { definitions } & 21\end{array}$

B Behaviour of the various terms in the intensity multiplier $\quad 22$

\section{Introduction}

The diffuse $\gamma$-ray background (DGRB) is, on angular scales larger than one degree, an isotropic radiation believed to be mostly of extragalactic origin. While it is established that several classes of astrophysical sources contribute to the DGRB (active galactic nuclei, star-forming galaxies, millisecond pulsars), its exact composition remains uncertain [1]. The DGRB is also one of the many targets for indirect dark matter (DM) searches [1, 2], via annihilation or decay of DM at Galactic and cosmological scales.

Indirect signs of annihilating DM due to secondary $\gamma$-radiation were first considered at the end of the 1970s in the context of diffuse astrophysical $\gamma$-ray emissions [3, 4]. It was concluded that the $\gamma$-ray signal from extragalactic DM was negligible compared to the one from Galactic DM, as has been confirmed by several subsequent studies (e.g., [5, 6]). Twenty years later, the calculation was revisited $[7,8]$ based on an improved understanding of structure formation in the $\Lambda \mathrm{CDM}$ cosmological paradigm: contrarily to previous estimates, the DGRB was found to be a promising entity to probe for signatures of DM annihilation. Many efforts followed to refine this calculation (see [1] for a comprehensive list of references), or to go beyond the simple average calculation to increase the sensitivity to the DM signal against astrophysical backgrounds (via the photon distribution function [9], searching for a small-scale anisotropy in the DGRB via auto-correlations [10] or cross-correlations with galaxy catalogues [11]). Based on these calculations and the analysis of four years of FermiLAT data [12,13], several authors [13-16] recently concluded that the limits on dark matter candidates derived from the DGRB are competitive with the best constraints on DM set by dwarf spheroidal galaxies [17, 18], and that they are currently the best limits set by Fermi-LAT data at TeV masses. 
In consequence, it is crucial to understand and reduce as much as possible the modelling uncertainties when estimating this exotic extragalactic contribution to the DGRB. These uncertainties come from our limited knowledge of several input ingredients (mass function of the DM halos, their mass concentration and density profile, etc.), as already discussed in the literature. Most of these studies were performed in the framework of the halo model description in real space $[8,12,13,19-24]$, with the exception of $[25,26]$ who proposed an approach based on the non-linear matter power spectrum in Fourier space. The two approaches are complementary, with slightly different uncertainties, and they were compared and found in reasonable agreement in [13]. Overall, these authors argue for a factor 20 uncertainty on the cosmological-induced DM signal.

This work is based on halo model descriptions relying on recent results for the halo mass function $[27,28]$ and the mass-concentration relation $[29,30]$ to provide an updated value for the cosmological signal from DM annihilations. Indeed, recent halo mass functions are based on updated cosmological parameters, more in line with the Planck cosmology [31]; recent mass-concentration relations also better account for the connection between the halo accretion history and formation time, allowing for an improved description of the concentration, valid in principle up to any redshift. For comparison purposes, we also consider other descriptions $[32,33]$ that have been used in previous estimates of the extragalactic DM contribution to the DGRB.

Our main goal is to bracket more closely the $\gamma$-ray signal uncertainties and to rank the various sources of uncertainties. It is useful to split the $\gamma$-ray signal into (i) a robustly determined lower-limit contribution from high-mass halos only $\left(M \gtrsim 10^{10} \mathrm{M}_{\odot}\right)$, and (ii) a larger but more uncertain contribution from less massive halos; the latter could actually be dominant in CDM scenarios in which very low-mass subhalos survive, but largely bf negligible for some classes of interacting DM (IDM). The many ingredients and calculations presented below will be available in a forthcoming release of the CLUMPY code ${ }^{1}$. In the context of the continuous increase of Fermi-LAT data (see the sensitivity projections for DM targets [42]), it is important to be able to easily repeat and improve on the calculation, as soon as more robust ingredients become available. In particular, the absolute DM intensity level also determines the feasibility of photon statistic or spatial cross-correlation studies in search for DM.

The paper is organised as follows: in section 2, we recall the formalism to calculate the contribution of extragalactic annihilating DM to the DGRB. The $\gamma$-ray signal in the context of collisionless CDM scenarios is presented in section 3, where we discuss the lower limit of this contribution, its uncertainties, and the signal dependence on low-mass extrapolations of the mass function. We discuss the CDM results in section 4, also commenting on self-interacting DM (SIDM), before concluding in section 5.

\footnotetext{
${ }^{1}$ CLUMPY $[34,35]$ is a public code for the calculation of annihilating or decaying DM in various DM targets. It was previously used for dwarf spheroidal galaxies [36-38], galaxy clusters [39, 40], and Galactic dark clumps [41]. It is being developed for the extragalactic emissions (average, distribution function, and angular power spectrum).
} 


\section{$2 \quad$ Modelling the $\gamma$-ray emission from extragalactic DM halos}

The extragalactic differential $\gamma$-ray intensity of annihilating DM, averaged over the whole sky, is given by

$I\left(E_{\gamma}\right)=\left\langle\frac{\mathrm{d} \Phi}{\mathrm{d} E_{\gamma} \mathrm{d} \Omega}\right\rangle_{\text {sky }}=\left.\frac{\bar{\varrho}_{\mathrm{DM}, 0}^{2}\langle\sigma v\rangle}{8 \pi m_{\chi}^{2}} \int_{0}^{z_{\max }} c \mathrm{~d} z \frac{(1+z)^{3}}{H(z)}\left\langle\delta^{2}(z)\right\rangle \frac{\mathrm{d} N_{\text {Source }}^{\gamma}}{\mathrm{d} E_{\mathrm{e}}}\right|_{E_{\mathrm{e}}=(1+z) E_{\gamma}} \times e^{-\tau\left(z, E_{\gamma}\right)}$,

with $E_{\gamma}$ the observed energy, $\Phi$ the flux, $\mathrm{d} \Omega$ the elementary solid angle, $c$ the speed of light, $\bar{\varrho}_{\mathrm{DM}, 0}$ the DM density of the Universe today, and $H(z)$ the Hubble constant at redshift $z$. The remaining quantities in the equation are related to four important ingredients of the calculation, as described below.

Properties of the DM candidate. A DM candidate is described by its mass $m_{\chi}$, the velocity averaged annihilation cross section $\langle\sigma v\rangle$, and $\mathrm{d} N_{\text {source }}^{\gamma} / \mathrm{d} E_{\mathrm{e}}$ the differential $\gamma$-ray yield per annihilation - the yield must be evaluated at $E_{e}=(1+z) E_{\gamma}$ to get a photon at $E_{\gamma}$ today. Throughout this paper, we assume a neutralino-like Majorana DM candidate. ${ }^{2}$ We rely on the CLUMPY implementation [35] of PPPC4DMID [43] to calculate the yield for various final states, e.g., $b \bar{b}$ quarks or $\tau^{+} \tau^{-}$leptons, and only consider prompt $\gamma$-rays produced in the hadronization and decay cascades after the DM particles' self-annihilation. In principle, the source term should also account for inverse Compton (IC) upscattered photons of the Cosmic Microwave Background $(\mathrm{CMB})$ off DM-induced electrons at relativistic energies $E_{e}$. These upscattered CMB photons have characteristic energies of $E_{\mathrm{IC}} \approx 3.4 \times 10^{-2}(1+z)\left(E_{e} /(100 \mathrm{GeV})\right)^{2} \mathrm{GeV}$ [44] and their spectrum peaks at lower energies compared to the prompt $\gamma$-ray emission. The relative importance of IC emission w.r.t. the prompt $\gamma$-rays and its energy dependence as a function of the final state and DM mass are illustrated in figure 1 of [44]. In this study, we are mostly interested in the high-energy end of the $\gamma$-ray spectrum and the relative uncertainty on the signal, which is independent of the low-energy spectral shape. For this reason, we do not include the contribution from IC scattering here, but remind that it could change the DM $\gamma$-ray spectra shown in later plots at energies $E_{\gamma} \lesssim 10^{-2} m_{\chi}$.

Transparency of the Universe to $\gamma$-rays. The term $\tau\left(z, E_{\gamma}\right)$ in Eq. (2.1) is the optical depth due to $e^{+} e^{-}$pair production of $\gamma$-rays on the infrared and optical extragalactic background light (EBL) and on the cosmic microwave background (CMB). This encodes the increasing attenuation of the extragalactic $\gamma$-rays from $\mathrm{GeV}$ to $\mathrm{TeV}$ energies and beyond. The values of the optical depth at various $z$ and $E_{\gamma}$ and its overall impact on the $\gamma$-ray spectrum are further discussed in $\S 3.1$.

Intensity multiplier from DM distribution. Density fluctuations in the early Universe give rise to an inhomogeneous distribution of $\mathrm{DM}, \varrho_{\mathrm{DM}}(\Omega, z)=\delta(\Omega, z) \times \bar{\varrho}_{\mathrm{DM}}(z)$. The inhomogeneous mass distribution boosts the rate of DM annihilations, expressed by the intensity multiplier $\left\langle\delta^{2}\right\rangle=1+\operatorname{Var}(\delta)$ in Eq. (2.1). For smoothly distributed DM, $\delta \equiv 1$, $\operatorname{Var}(\delta)=0$ and $\left\langle\delta^{2}\right\rangle=1$, whereas for a high density contrast, $\left\langle\delta^{2}\right\rangle \approx \operatorname{Var}(\delta) \gg 1$. The contribution to the $\gamma$-ray intensity from the inhomogeneous Universe dominates at $z \lesssim 50$ $[22,29]$ and we only consider this contribution in this work (see also figure 10 in appendix B).

The quantity $\left\langle\delta^{2}(z)\right\rangle$ can either be directly computed from the nonlinear matter power spectrum $P_{\mathrm{nl}}(k, z)[25,26]$, or from using the halo model approach that we adopt here (as,

\footnotetext{
${ }^{2}$ For a Dirac particle, the factor $8 \pi$ in the denominator of Eq. (2.1) would be $16 \pi$.
} 
e.g., followed by $[8,24,45])$. In the halo model setup, the intensity multiplier from the inhomogeneous Universe is written as

$$
\left\langle\delta^{2}(z)\right\rangle=\frac{1}{\bar{\varrho}_{\mathrm{m}, 0}^{2}} \int \mathrm{d} M \frac{\mathrm{d} n}{\mathrm{~d} M}(M, z) \times \mathcal{L}(M, z),
$$

with today's mean total matter density $\bar{\varrho}_{\mathrm{m}, 0}$, the halo mass function $\mathrm{d} n / \mathrm{d} M$ (i.e. the comoving number density of halos of mass $M$ at redshift $z$ ), and the comoving one-halo luminosity, $\mathcal{L}(M, z)$; these terms are described in the following way:

- The halo mass function $\mathrm{d} n / \mathrm{d} M$ is usually expressed in terms of the variance $\sigma$ of the density fluctuations in the linear regime and of the multiplicity function $f(\sigma, z)$ that encodes nonlinear structure formation,

$$
\frac{\mathrm{d} n}{\mathrm{~d} M}(M, z)=f(\sigma, z) \frac{\bar{\varrho}_{\mathrm{m}, 0}}{M} \frac{\mathrm{d} \ln \sigma^{-1}}{\mathrm{~d} M}
$$

The density variance $\sigma$ is a function of the linear matter power spectrum, $P_{\text {lin }}(k, z=0)$, according to

$$
\sigma^{2}(M, z)=\frac{D(z)^{2}}{2 \pi^{2}} \int P_{\operatorname{lin}}(k, z=0) \widehat{W}^{2}(k R) k^{2} \mathrm{~d} k,
$$

where a collapsing region of comoving radius $R=\left[3 M /\left(4 \pi \bar{\varrho}_{\mathrm{m}, 0}\right)\right]^{1 / 3}$ containing the mass $M$ is defined by a spherical top-hat window $W$, with $\widehat{W}(k R)=3(k R)^{-3}[\sin (k R)-$ $k R \cos (k R)]$ in Fourier space. We use the CLASS code [46] to compute $P_{\text {lin }}(k, z=0)$ and we evolve $\sigma(M, z)^{2}$ to higher redshifts via the growth factor $D(z)=g(z) / g(z=0)$ with [47]

$$
g(z)=\frac{5}{2} \Omega_{\mathrm{m}, 0} H_{0}^{2} \times H(z) \int_{z}^{\infty} \frac{1+z^{\prime}}{H^{3}\left(z^{\prime}\right)} \mathrm{d} z^{\prime},
$$

where $\Omega_{\mathrm{m}, 0}=\frac{8 \pi G}{3} H_{0}^{-2} \times \bar{\varrho}_{\mathrm{m}, 0}$ and $G$ is the gravitational constant.

- The comoving one-halo luminosity,

$$
\mathcal{L}\left(M_{\Delta}, z\right)=\int \mathrm{d} V \rho_{\text {halo }}^{2}=4 \pi \int_{0}^{R_{\Delta}} \mathrm{d} r r^{2} \rho_{\text {halo }}^{2},
$$

is a function of the halo mass $M_{\Delta}$, a given halo density profile ${ }^{3} \rho_{\text {halo }}\left(r ; \rho_{-2}, r_{-2}\right)$, and a mass-concentration relation

$$
c_{\Delta}\left(M_{\Delta}, z\right):=\frac{R_{\Delta}}{r_{-2}}
$$

that is required to determine the normalisation of the profile given the halo mass. The subscript $\Delta$ denotes that a halo mass $M_{\Delta}$ is connected to its size, $R_{\Delta}$, via the relation

$$
R_{\Delta}\left(M_{\Delta}, z\right)=\left(\frac{3 M_{\Delta}}{4 \pi \times \Delta(z) \times \varrho_{\mathrm{c}}(z)}\right)^{1 / 3} \times(1+z),
$$

where $\varrho_{\mathrm{c}}=\frac{3}{8 \pi G} H^{2}(z)$ is the critical density of the Universe. While numerical simulations provide reasonably precise and scale-invariant density profiles $\rho_{\text {halo }}$, the halo mass depends on the definition used for the halo outer bound [e.g., 48].

\footnotetext{
${ }^{3}$ The radius $r_{-2}$ is defined as $\mathrm{d} \log \rho_{\text {halo }} /\left.\mathrm{d} \log r\right|_{r=r_{-2}}=-2$, and $\rho_{-2}:=\rho_{\text {halo }}\left(r=r_{-2}\right)$.
} 
Redshift range. In principle, all radiation from DM relic annihilation after the recombination era would contribute to today's observed intensity, Eq. (2.1). However, the cosmic $\gamma$-ray horizon for $E_{\gamma} \sim 100 \mathrm{GeV}$ photons due to pair-production on the EBL lies at $z \sim 1$ [49] and at lower redshifts for higher energies. Conversely, at the lowest energies, $E_{\gamma} \lesssim 20 \mathrm{GeV}$, the Universe is mostly transparent to $\gamma$-rays and radiation from high redshifts could significantly contribute to the intensity. In the remainder of the paper, we perform the numerical integration of the $\gamma$-ray intensity up to $z_{\max }=10$, corresponding to the highest redshift regime for which calculations of the DM distribution and models of the EBL are available. ${ }^{4}$

\section{Exotic $\gamma$-ray intensity in the collisionless CDM paradigm}

Almost all ingredients above are related to DM halo properties, which are mostly studied by means of numerical simulations at cosmological scales [50]. Their finite mass resolution does not generally allow the characterisation of the halo population below $M \sim 10^{10} \mathrm{M}_{\odot}$, while the smallest DM protohalos can possibly form down to $10^{-12} \mathrm{M}_{\odot}[51-53]$ - the exact mass cut-off is related to the properties and kinetic decoupling of the DM candidate. This is an issue as this low-mass population may be responsible for a large part of the signal, and accounting for it is the largest source of uncertainty on the $\gamma$-ray signal (see $\S 3.2$ ). Providing a universal description of the halo properties at all scales and all redshifts is further complicated by the various origins of the physics processes and environments in which these halos form and evolve [54].

The halo properties used in our calculation are taken from the most advanced results from the literature, favouring those attempting to provide a universal description over all scales and epochs. The various results obtained by different groups allow us to define a range of values for these ingredients. Propagated to the intensity calculation, they provide reasonable and hopefully realistic uncertainties for the $\gamma$-ray emission estimate. The reference parameters and the range or configurations used are gathered in table 1; the corresponding results and discussions are detailed in $\S 3.1$ and $\S 3.2$.

\subsection{Contribution $I_{0}$ from high-mass halos $\left(M \geq 10^{10} \mathrm{M}_{\odot}\right)$ : a robust lower bound}

In this first result section, we focus on the safe mass range $M \geq 10^{10} \mathrm{M}_{\odot}$, where constraints exist from both numerical simulations and observations. Also, we do not take into account substructures in these halos. The derived intensity will provide a lower limit on the DM contribution to the DGRB.

The top third of table 1 (denoted reference intensity) and figure 1 summarise the results that will be discussed in this section. In this figure, we present the exotic extragalactic intensity for $\mathrm{DM}$ candidates of $100 \mathrm{GeV}$ (left column) and $10 \mathrm{TeV}$ (right column). While a $100 \mathrm{GeV}$ particle corresponds to the canonical mass scale of a generic weakly interacting massive particle (WIMP), $m_{\chi}=10 \mathrm{TeV}$ marks the regime of the largest expected WIMP masses [64]. Additionally, these two candidates allow us to explore regimes of weak and strong EBL absorption of remote $\gamma$-rays. For illustrative purpose, we also always display results for the $b \bar{b}$ (green) and the harder $\tau^{+} \tau^{-}$(magenta) annihilation channels. The top row in figure 1 corresponds to the intensity from the reference model, while lower panels show the deviation obtained by changing the ingredients of the default configuration; this is discussed in the following paragraphs.

\footnotetext{
${ }^{4}$ Before the formation of the first stars, the only low-energy radiation background in the Universe was the CMB.
} 


\begin{tabular}{|c|c|c|c|}
\hline \multicolumn{4}{|c|}{$\begin{array}{l}\text { Reference intensity: } I_{0} \\
\left(M \geq 10^{10} \mathrm{M}_{\odot}, \text { no subhalos }\right)\end{array}$} \\
\hline Physics properties & Reference $I_{0}$ & Variations $I_{0, \text { var }}$ & $\left|I_{0}-I_{0, \operatorname{var}}\right| / I_{0}$ \\
\hline Halo mass function ${ }^{\dagger}$ & $\mathrm{R} 16[28]$ & T08 [32], B16 [55] & $\lesssim 40 \%$ \\
\hline Density profile $\rho_{\text {halo }}$ & $\alpha_{\mathrm{E}}=0.17$ & $\alpha_{\mathrm{E}}=0.15, \alpha_{\mathrm{E}}=0.22, \mathrm{NFW}$ & $\lesssim 20 \%$ \\
\hline$c_{\Delta}\left(M_{\Delta}\right)$ relation $^{\ddagger}$ & $\mathrm{C} 15[29]$ & $\mathrm{L} 16[30], \mathrm{C} 15-\sigma_{c}=0.2,(\mathrm{~S} 14)$ & $\lesssim 10 \%$ \\
\hline Cosmology $\left(h, \Omega_{i}, P_{k}\right)^{\S}$ & Planck-R16 [28] & WMAP7 [56], (WMAP-T08) & $\lesssim 10 \%$ \\
\hline Overdensity definition & $\Delta_{\text {vir }}(3.3)$ & $\Delta_{\mathrm{c}}(3.1)$ or $\Delta_{\mathrm{m}}(3.2)=200$ & $\lesssim 5 \%$ \\
\hline EBL model ${ }^{\star}$ & $\mathrm{I} 13[57]$ & F08 [58], D11 [59], G12 [60] & $\lesssim 5-40 \%$ \\
\hline \multicolumn{4}{|c|}{$\begin{array}{l}\text { Total CDM contribution: } I_{l} \text { (extrapolation to low masses) } \\
\qquad\left(M \geq M_{\min }, \text { no subhalos }\right)\end{array}$} \\
\hline Field halo properties & \multicolumn{2}{|c|}{ Values (default in bold) } & $I_{l} / I_{0}(\simeq 5)$ \\
\hline Slope of $\mathrm{d} n / \mathrm{d} M, \alpha_{M}$ & \multicolumn{2}{|c|}{$1.85, \mathbf{1 . 9}, 1.95$} & $\sim 4-14$ \\
\hline Minimal mass $M_{\min }$ & \multicolumn{2}{|c|}{$10^{-12}, \mathbf{1 0}^{-\mathbf{6}}, 10^{-3} \mathrm{M}_{\odot}$} & $\sim 4-8$ \\
\hline Density profile $\rho_{\text {halo }}$ & \multicolumn{2}{|c|}{$\alpha_{\mathrm{E}}=0.15, \mathbf{0 . 1 7}, 0.22, \mathrm{NFW}$, Ishiyama $[61]$} & $\sim 4-8$ \\
\hline$c_{\Delta}\left(M_{\Delta}\right)$ relation ${ }^{\ddagger}$ & \multicolumn{2}{|c|}{ C15 [29], L16 [30], (S14 [33]) } & $\sim 3-8$ \\
\hline \multicolumn{4}{|c|}{$\begin{array}{l}\text {. including boost from subhalos: } I_{\mathrm{b}} \\
\quad\left(m \geq m_{\min } \text { with } m_{\min } \equiv M_{\min }\right)\end{array}$} \\
\hline (Sub-)halo properties & \multicolumn{2}{|c|}{ Values (default in bold) } & $I_{\mathrm{b}} / I_{l}(\simeq 1.5)$ \\
\hline Mass fraction $f_{\text {subs }}$ & \multicolumn{2}{|c|}{$10 \%, \mathbf{2 0} \%, 40 \%$} & $\sim 1.2-2.2$ \\
\hline Minimal mass $m_{\min }$ & \multicolumn{2}{|c|}{$10^{-12}, \mathbf{1 0}^{-\mathbf{6}}, 10^{-3} \mathrm{M}_{\odot}$} & $\sim 1.3-1.8$ \\
\hline$c_{\Delta}\left(M_{\Delta}\right)$ relation ${ }^{\ddagger}$ & \multicolumn{2}{|c|}{ C15 [29], L16 [30], (S14 [33]) } & $\sim 1.3-1.7$ \\
\hline Density profile $\rho_{\text {subhalo }}$ & \multicolumn{2}{|c|}{$\alpha_{\mathrm{E}}=0.15, \mathbf{0 . 1 7}, 0.22, \mathrm{NFW}$, Ishiyama $[61]$} & $\sim 1.3-1.7$ \\
\hline Slope of $\mathrm{d} P / \mathrm{d} m, \alpha_{m}$ & \multicolumn{2}{|c|}{$1.85,1.9,1.95$} & $\sim 1.4-1.7$ \\
\hline $\mathrm{d} P / \mathrm{d} V$ profile & \multicolumn{2}{|c|}{ Aquarius [62], Phœnix [63], $\propto \rho_{\text {host }}$} & $\sim 1.49-1.51$ \\
\hline
\end{tabular}

Table 1: Parameters and options used for the calculations of the intensity, Eq. (2.1). The table is organised in three blocks, starting from the high-mass halo contribution $I_{0}\left(M>10^{10} \mathrm{M}_{\odot}\right)$ discussed in $\S 3.1$, the all-mass halo contribution $I_{l}$ where extrapolations of the parameters are used in the low-mass range ( $\S 3.2 .1$ ), and finally $I_{\mathrm{b}}$ that accounts for substructures in the halos ( $\left.\S 3.2 .2\right)$. In more details, from top to bottom: (i) benchmark parameters, their alternatives, and induced uncertainties on the reference intensity $I_{0}$ (the models given in grey/parentheses are investigated but not included in the error budget - see discussion and figures 1, 4 and 5); (ii) parameters extrapolated to low masses, range of their values, and impact on the ratio $I_{l} / I_{0}$ (see figure 4); (iii) subhalo parameters, range of their values, and impact on the ratio $I_{\mathrm{b}} / I_{l}$ (see figure 5 ). Note that for (ii) and (iii) the bold values correspond to the default configuration used when varying one parameter at a time. Also, the minimal mass, the concentration $c_{\Delta}\left(M_{\Delta}\right)$, and halo profiles are always tied between field and subhalos.

Cosmology. The extragalactic exotic $\gamma$-ray intensity given by Eq. (2.1) depends on cosmology through today's mean DM density, the Hubble expansion rate and the halo mass function. The latter depends not only on the cosmology but also on the specific parame- 


\begin{tabular}{lcccccc}
\hline \hline & $h$ & $\Omega_{\mathrm{m}, 0}$ & $\Omega_{\mathrm{b}, 0}$ & $\Omega_{\Lambda, 0}$ & $\sigma_{8}$ & $n_{s}$ \\
\hline Planck-R16 & 0.678 & 0.307 & 0.048 & 0.693 & 0.829 & 0.96 \\
WMAP7 & 0.704 & 0.272 & 0.0456 & 0.728 & 0.809 & 0.963 \\
WMAP-T08 & 0.7 & 0.3 & 0.04 & 0.7 & 0.9 & 1 \\
\hline
\end{tabular}

Table 2: Cosmological parameter sets considered in this study with $h=H_{0} / 100 \times \mathrm{Mpc} \mathrm{s} \mathrm{km}^{-1}$, $\varrho_{i}(z=0)=\Omega_{i, 0} \times \varrho_{\mathrm{c}}(0), \sigma_{8}=\sigma\left(R=8 h^{-1} \mathrm{Mpc}\right)$, and $n_{\mathrm{s}}$ the spectral index of the primordial power spectrum. Planck $-\mathrm{R} 16$ corresponds to the Planck cosmology as implemented in the MultiDark-Planck and Bolshoi-Planck simulations [27] used by Rodríguez-Puebla et al. (2016, R16 [28]). The WMAP7 cosmology was implemented in the Magneticum simulations used by Bocquet et al. (2016, B16 [55]). Finally, WMAP-T08 corresponds to one of the WMAP1-3 cosmologies implemented in the simulations used by Tinker et al. (2008, T08 [32]).

ters of the multiplicity function that are fitted to results of numerical simulations. We first evaluate the impact of the cosmological parameters alone, using the sets of parameters given in table 2. The latter correspond to the underlying cosmologies of several simulations from which the mass functions discussed below have been derived. We use the Planck-R16 cosmology as our reference, and study the impact on the intensity when switching to the WMAP7 or WMAP-T08 cosmologies. The results are displayed in the second row of figure 1.

When using the WMAP-T08 cosmology, the effect can reach $\sim 50 \%$ for the $100 \mathrm{GeV}$ DM candidate; this is shown for illustrative purposes only, as this outdated set of parameters is ruled out by more recent estimates. Apart from this case, switching between Planck and WMAP7 cosmologies, the impact on the intensity remains a rather marginal $\sim 10 \%$ effect, and we only propagate this uncertainty to our total error budget. Note that the effect is even slightly smaller for the $10 \mathrm{TeV} \mathrm{DM}$ candidate (right) than for the $100 \mathrm{GeV}$ candidate (left). For $\mathrm{TeV}$ dark matter, the ratio to the reference intensity is also rather independent of the annihilation channel; at these high energies the EBL absorption is such that the $\gamma$-rays have a local origin only, i.e. their spectra are not redshifted and the ratio to the reference intensity is therefore the same for both channels. This behaviour will be present in all the cases we explore below.

Halo mass function/multiplicity function. We now turn to the choice of the parametrisation of the multiplicity function $f(\sigma, z)$ entering the calculation of the mass function $\mathrm{d} n / \mathrm{d} M$ according to Eq. (2.3). In the last fifteen years, the parametrisation of $f(\sigma, z)$ has evolved, following the improvements of cosmological simulations [50]. As mentioned above, for this work we select the recent Rodríguez-Puebla et al. parametrisation (R16, [28]) as our reference mass function. In order to bracket the modelling uncertainties, we also consider the widely-used Tinker et al. (T08, [32]) and the Bocquet et al. (B16, [55]) DM-only parametrisations. $^{5}$ In B16, the authors also provide results based on hydrodynamical simulations, including baryon feedback on structure formation. Using these results yield only a $10 \%$ difference on the exotic extragalactic intensity compared to the DM-only parametrisation, so we only consider the latter below.

The third and fourth rows in figure 1 show the impact of the choice of multiplicity function when (i) the Planck cosmology is used whatever the chosen multiplicity function or when (ii) a given $f(\sigma, z)$ is combined with the "right" cosmology, i.e. the one of the

\footnotetext{
${ }^{5}$ We validate our implementation of the cosmology and mass functions in the CLUMPY code from a successful comparison to the original publications, i.e. using the same underlying cosmology as the one the mass function was derived from (see table 2 and figure 7 in appendix B).
} 

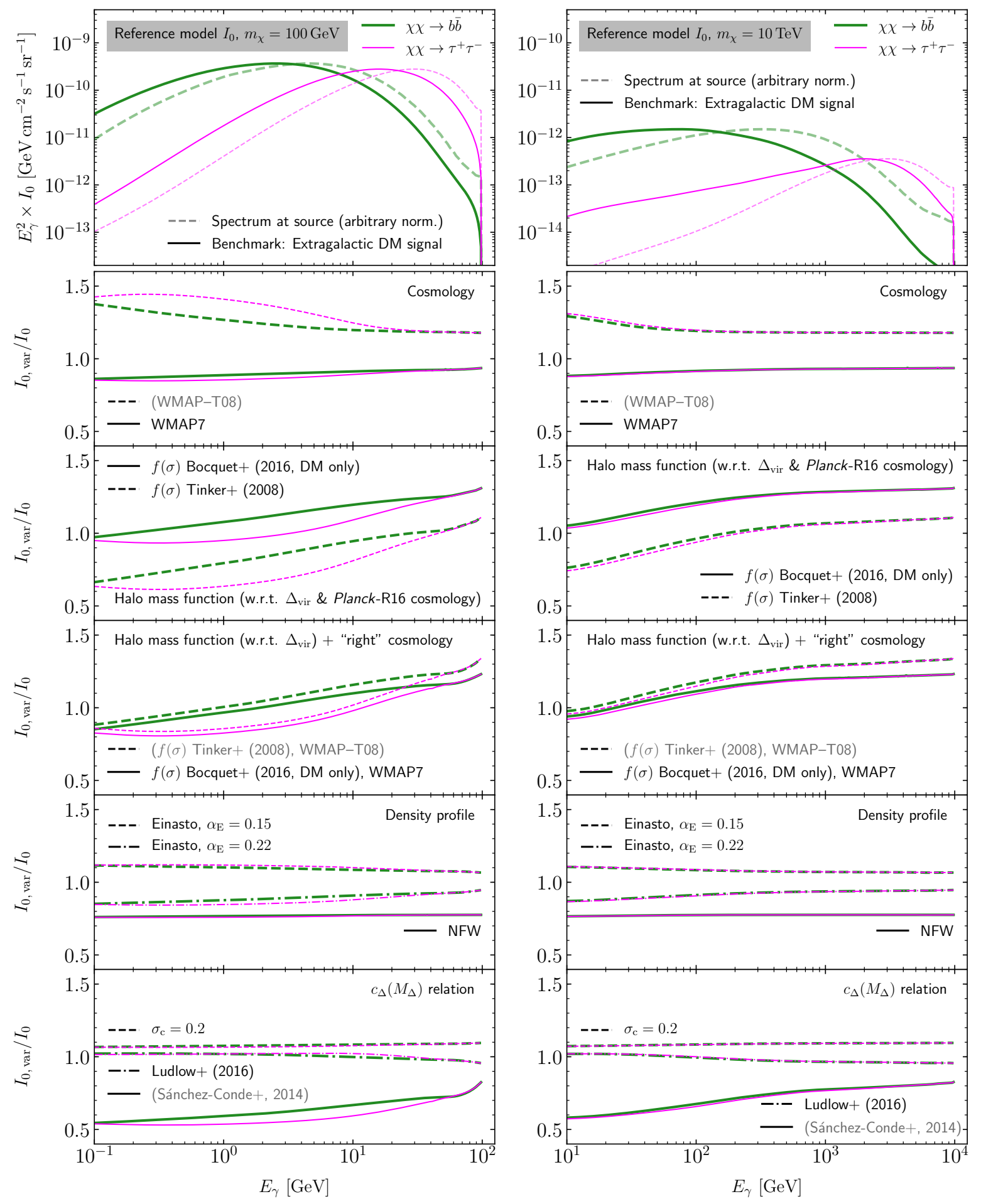

Figure 1: Top row: Reference intensity $I_{0}\left(M \geq 10^{10} \mathrm{M}_{\odot}\right.$, no subhalos $)$ for $\langle\sigma v\rangle=3 \times 10^{-26} \frac{\mathrm{cm}^{3}}{\mathrm{~s}}$ and a light $\left(100 \mathrm{GeV}\right.$, left) or heavy (10 TeV, right column) WIMP. Pure $\chi \chi \rightarrow b \bar{b}$ (green) and $\chi \chi \rightarrow \tau^{+} \tau^{-}$ (magenta curves) annihilation channels are shown. Dashed lines in the top row display the source spectra without EBL absorption and cosmological redshift. Lower rows: Ratios $I_{0}$ to variations $I_{0, \mathrm{var}}$. Bracketed models are excluded from the error budget. See table 1 and discussion in $\S 3.1$. 
simulations used to determine its parameters. In both cases, there is at most a $\sim 40 \%$ difference with respect to the reference intensity.

Overdensity definition. The relation between a halo size and its mass is given by Eq. (2.8) and depends on the overdensity quantity $\Delta(z)$. Various definitions are found in the literature, namely

$$
\begin{aligned}
& \Delta(z)=\text { const. }=: \Delta_{\mathrm{c}} \\
& \Delta(z)=\text { const. } \times \Omega_{\mathrm{m}}(z)=: \Delta_{\mathrm{m}} \times \Omega_{\mathrm{m}}(z) \\
& \left.\Delta(z)=18 \pi^{2}+82\left[\Omega_{\mathrm{m}}(z)-1\right)\right]-39\left[\Omega_{\mathrm{m}}(z)-1\right]^{2}=: \Delta_{\text {vir }} \quad([65], \text { for a flat Universe }) .
\end{aligned}
$$

Halo mass functions are generally given for several values of $\Delta(z)$, common choices being $\Delta_{\mathrm{c}, \mathrm{m}}=200$. Although not shown in figure 1, we also explore how the results are affected by this choice. The methodology to convert the halo mass between different $\Delta(z)$ is described in appendix A. Using $\Delta_{\text {vir }}$ as the reference, we find a very marginal effect of $\lesssim 5 \%$ when switching to $\Delta_{\mathrm{c}}=200$ or $\Delta_{\mathrm{m}}=200$.

Halo density profile. The halo profile enters into the intrinsic luminosity term Eq. (2.6). Two standard, spherically-symmetric ${ }^{6}$ parametrisations of DM profiles are the NFW [68] and Einasto [69] profiles,

$$
\begin{aligned}
\rho^{\mathrm{NFW}}(r) & =\frac{4 \rho_{-2}}{\left(r / r_{-2}\right)\left(1+r / r_{-2}\right)^{2}}, \\
\rho^{\text {Einasto }}(r) & =\rho_{-2} \exp \left(-\frac{2}{\alpha_{\mathrm{E}}}\left[\left(\frac{r}{r_{-2}}\right)^{\alpha_{\mathrm{E}}}-1\right]\right),
\end{aligned}
$$

with $r_{-2}$ the radius for which the logarithmic slope equals -2 , and $\rho_{-2}=\rho\left(r_{-2}\right)$ the normalisation. The NFW profile has an inner slope of -1 , whereas the Einasto profile logarithmically tends to a flat profile, with the sharpness of the inner profile controlled by $\alpha_{\mathrm{E}}$; the smaller $\alpha_{\mathrm{E}}$, the steeper the slope.

The Einasto parametrisation has been found to better fit DM halos than the NFW profile, in both DM-only [62, 70, 71] and hydrodynamical simulations [72], and at various scales. A large suite of Milky-Way size simulated halos $\left(M \sim 10^{12} \mathrm{M}_{\odot}\right)$ obtained $\alpha_{\mathrm{E}} \approx$ $0.17 \pm 0.02$ [73], in agreement with the results of [62]. However, this slope is not universal, and dedicated DM-only simulations found that $\alpha_{\mathrm{E}}$ increases with both the mass and redshift [71, 74, 75]. In particular between $M=10^{11} \mathrm{M}_{\odot}$ and $M=10^{16} \mathrm{M}_{\odot}$, [71] finds that $\alpha_{\mathrm{E}}$ increases from 0.16 to 0.22 . At these high masses, the hydrodynamical feedback from active galactic nuclei in the halo centers can affect the profile [76]. From the observational point of view, lensing constraints have found $0.17<\alpha_{\mathrm{E}}<0.21$ for halos with $M \sim 10^{15} \mathrm{M}_{\odot}$ [77], whereas X-ray data analyses found somewhat a larger range of values with $0.14<\alpha<0.26$ [78] or $\alpha \approx 0.29$ [79].

For our reference calculation of $I_{0}$, we assume an Einasto profile of index $\alpha_{\mathrm{E}}=0.17$. This value is more representative of Milky-way like halos and small groups than galaxy clusters, but the most massive ones are not numerous and are subordinate in the $\gamma$-ray signal (see the sharp decrease of the mass function above $10^{14} \mathrm{M}_{\odot}$ at $z=0$ in figure 3 ). We do not include any mass and redshift dependence in $\alpha_{\mathrm{E}}$, but $\alpha_{\mathrm{E}}$ is varied from 0.15 to 0.22 to encompass the

\footnotetext{
${ }^{6} \mathrm{DM}$ halos are not spherical, the more massive halos being more triaxial [e.g., 66, 67]. However, as we average on large numbers of halos and orientations, we do not expect triaxiality to impact the results.
} 
possible values obtained in the simulations and the data. The impact on the $\gamma$-ray intensity is shown in the fifth row of figure 1, where a $10-15 \%$ effect is observed. We also show the comparison with a NFW profile, which is $\sim 20 \%$ below the Einasto reference profile, but close to $\alpha_{\mathrm{E}}=0.22$. This is both in agreement with the fact that Einasto profiles with this index are close to NFW profiles, and the fact that despite they asymptotically flatten, they give a larger $\gamma$-ray signal; this is because they produce larger densities than NFW profiles of same mass and mass concentration in regions which dominate the signal from annihilation.

Mass-concentration-redshift parametrisation $c(M, z)$. Given a halo mass, a profile (Einasto or NFW), and a $\Delta$ definition, the mass-concentration relation Eq. (2.7) fully determines the structural parameters $r_{-2}$ and $\rho_{-2}$ in Eq. (3.4) and (3.5). This relation reflects the mass dependence of halo formation times, with less concentrated halos at earlier times. Early studies proposed a redshift dependence of $c(M, z) \propto(1+z)^{-1}[80,81]$, but a milder dependence was obtained in subsequent calculations for high-mass halos [82]. Several empirical models have been proposed since, taking advantage of the connections between halo mass profiles and the main progenitor mass accretion history.

We rely here on three models [29, 30, 33], namely S14 (Sánchez-Conde \& Prada, 2014), C15 (Correa et al., 2015), and L16 (Ludlow et al., 2016). The domain of validity of these models encompasses a large mass range, providing a consistent picture when extrapolating the mass function down to the lower masses (see $\S 3.2$ ). Moreover, the recent works by C15 and L16 include a dedicated redshift dependence study of the mass-concentration, which makes them appealing for the calculation of the $\gamma$-ray emission from far-away DM structures. While the S14 model was obtained for $z=0$ and is extended to higher redshifts by a $(1+z)^{-1}$ scaling, C15 and L16 found a milder evolution: also, S14 shows an upturn for the highest masses contrarily to the other two (see the comparison in figure 8 in appendix B). Whether this upturn is real or a selection bias in numerical simulations is still under discussion $[27,30] .{ }^{7}$ At the galaxy cluster scale, the $c(M, z)$ from simulations has been found in agreement with Xray and weak-lensing constraints, $c_{\mathrm{vir}} \sim 3-6$ [83], with a log-normal distribution of intrinsic scatter $\sigma_{c} \sim 0.12-0.22[79,83-85]$ also consistent with results from numerical simulations $[33,86]$. Observations up to $z \sim 1.2$ have shown no obvious redshift evolution [84], but more data are required to be more conclusive.

The C15 concentration is used as reference. The last panel of figure 1 shows the ratio of the $\gamma$-ray intensity to this reference for different concentration choices, using S14, L16, or applying a log-normal scatter to the $\mathrm{C} 15$ model $\left(\mathrm{C} 15-\sigma_{c}\right)$. The options L16 and $\mathrm{C} 15-\sigma_{c}$ give results within $10 \%$ of the reference. The $\gamma$-ray intensity from S14 is markedly below (up to $50 \%$ for the lowest energies), however, this difference is ascribed to the $(1+z)^{-1}$ evolution. Therefore, we do not consider the S14 model for our overall error budget and suggest a lower uncertainty on the intensity $I_{0}$ of not more than $10 \%$.

EBL absorption. Intergalactic low-energy radiation fields absorb high-energy $\gamma$-rays via production of $e^{+} e^{-}$pairs. The intensity of the extragalactic infrared- and optical background light can either directly be estimated by photometric measurements, integration over deepexposure galaxy counts, or indirectly by VHE observations of distant blazars (see [87] for a recent review). While different methods and measurements are hampered by different uncertainties and are able to give lower (galaxy counts) or upper (indirect $\gamma$-ray measurements)

\footnotetext{
${ }^{7}$ While such an upturn is generally expected to provide a minor contribution to the $\gamma$-ray signal as the number of very massive halos is strongly suppressed at high masses, it involves using the corresponding concentration relation beyond its fitting range.
} 

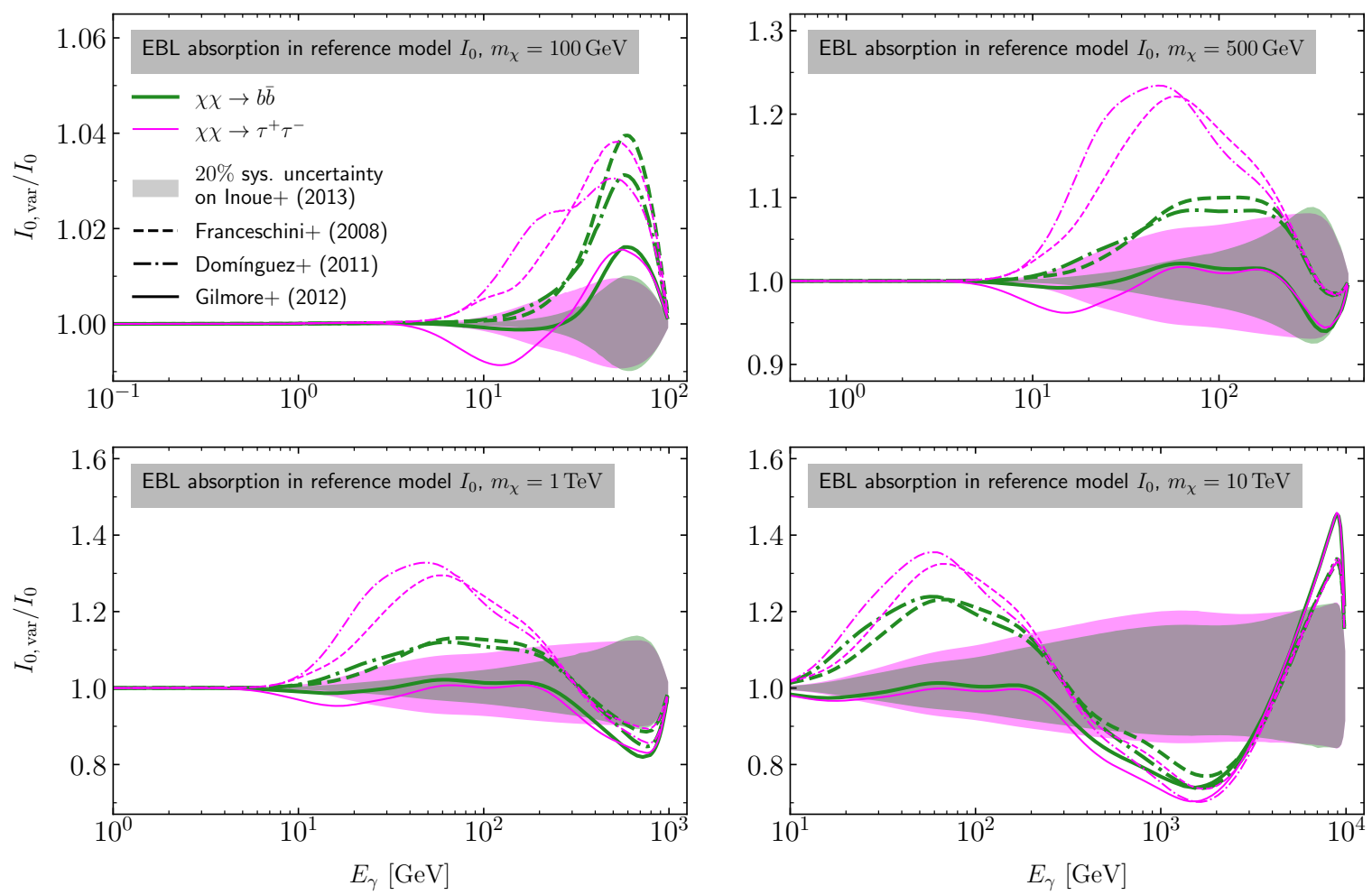

Figure 2: Uncertainty of the EBL extinction factor $\tau\left(E_{\gamma}, z\right)$ for four different WIMP masses between $100 \mathrm{GeV}$ and $10 \mathrm{TeV}$ and pure $\chi \chi \rightarrow b \bar{b}$ (green) and $\chi \chi \rightarrow \tau^{+} \tau^{-}$(magenta) annihilation channels. The shaded areas indicate a $20 \%$ systematic uncertainty on $\tau\left(E_{\gamma}, z\right)$ in the reference model [57].

limits on the EBL density, they mostly agree in determining the spectral EBL energy density at low redshifts, $z<2$; the largest uncertainties are in the far-infrared, where zodiacal light is dominant and astronomical observation intricate [88]. They significantly differ at redshifts $z>2$, caused by different extrapolations and evolution of the measurements into the past. For this work, we apply as default the model from [57], I13 (Inoue et al., 2013), where they attempt for the first time to consistently calculate, using semi-analytical models of hierarchical structure formation of dark and baryonic matter, the EBL density at redshifts back to the epoch of reionization. This allows us to integrate Eq. (2.1) up to $z_{\max }=10$. As their modelling predicts a factor $\sim 2$ lower $\gamma$-ray attenuation for $E_{\mathrm{e}} \gtrsim 400 \mathrm{GeV} \gamma$-rays compared to previous calculations, we compare their estimation of the cosmic $\gamma$-ray opacity with the classical, more data-driven models by [59] (D11, Domínguez et al., 2011) based on observed galaxy populations up $z=1$ (and their evolution into the past to $z=2$ ), the backwardevolution model from [58] providing EBL attenuations up to $z=4$ (F08, Franceschini et al., 2008), and the semi-analytic forward-evolution model by [60] up to $z=6$ (G12, Gilmore et al., 2012). At higher redshifts, we extrapolate their models by a power-law, however, this only affects the lowest energies $E_{\gamma} \lesssim 20 \mathrm{GeV}\left(E_{\gamma} \lesssim 10 \mathrm{GeV}\right)$, for which the Universe is transparent enough to $\gamma$-rays emitted at $z \gtrsim 2(z \gtrsim 4)$.

In figure 2, we compare results from these four models for four different DM masses. As has been stated by the authors themselves, at low energies, the model I13 agrees with G12 and predicts a larger attenuation than F08 and D11, while it significantly differs from all compared 
models at energies above $E_{\gamma} \gtrsim 200 \mathrm{GeV}$. For a better assessment of the discrepancy, we also show the range of a $20 \%$ systematic uncertainty on the attenuation factor $\tau$ in the I13 model. Overall, we find that the uncertainty from different models of the EBL increases with the $\gamma$-ray energy, from a marginal discrepancy at $E_{\gamma} \lesssim 50 \mathrm{GeV}$ up to a $40 \%$ uncertainty at the highest energies, where the absorption is strongest. In general, the uncertainty is larger for a hard annihilation channel like the $\chi \chi \rightarrow \tau^{+} \tau^{-}$case, with a larger relative amount of photons emitted at the high-energy end of the spectrum.

\subsection{Including low-mass halos and subhalos}

\subsubsection{Extrapolation of the mass function}

Cosmological simulations within the $\Lambda \mathrm{CDM}$ paradigm only determine halo structure down to halo masses of $M \gtrsim 10^{10} \mathrm{M}_{\odot}$. The number density of $\mathrm{DM}$ halos below the resolution limit is largely unknown, with however a major impact on the overall $\gamma$-ray intensity from DM annihilations. Overall, three quantities govern the $\gamma$-ray emission from small-scale DM structures: the number density of halos below the resolution limit, their minimal mass, and a possible higher mass concentration of lighter halos, which may further enhance the DM annihilation process.

The first row of figure 4 shows, for the reference configuration (see table 1), the intensity accounting for the extrapolated low-mass field halos, $I_{l}$, compared to the reference calculation $I_{0}$. DM halos below $10^{10} \mathrm{M}_{\odot}$ dominate the intensity with $I_{l} / I_{0} \sim 5$; the contribution of various halo masses and redshifts are explicit in the right panel of figure 9 in appendix B.

Power-law mass function extrapolation. For scale-invariant primordial perturbations, the matter power spectrum in a collisionless CDM paradigm approaches $P_{\text {lin }}(k) \propto k^{-3} \times \ln ^{2}(k)$ for $k \rightarrow \infty$ [26], and Press-Schechter theory predicts a corresponding power-law scaling of the mass function,

$$
\frac{\mathrm{d} n}{\mathrm{~d} M} \propto M^{-\alpha_{M}} \quad \text { for } M \rightarrow 0,
$$

with $\alpha_{M}=2$ [89]. We therefore adopt Eq. (3.6) to smoothly extrapolate the mass function below the minimal mass of our reference model, i.e. for $M<10^{10} \mathrm{M}_{\odot}$. However, assuming $\alpha_{M}=2$ and the mass functions listed in table 1 , the comoving density of mass contained in halos, $\varrho_{\text {halos }}(z)=\int_{M_{\min }}^{\infty} M \mathrm{~d} n(z) / \mathrm{d} M \mathrm{~d} M$, exceeds the mean density $\bar{\varrho}_{\mathrm{m}, 0}$ with $M_{\min }$ far above the natural mass cut-off scale (discussed in the next paragraph). ${ }^{8}$ On the other hand, we obtain $\varrho_{\text {halos }}\left(M_{\min }=10^{-12} \mathrm{M}_{\odot}\right) \leq \bar{\varrho}_{\mathrm{m}, 0}$ with $\alpha_{M}=1.95$ for all considered cosmologies and mass functions. By this argument we choose $\alpha_{M}=1.95$ as an upper bound to consistently explore different minimal particle-physics motivated cut-off scales. As a lower bound on $\alpha_{M}$, we select $\alpha_{M}=1.85$, which is the typical low-mass asymptotic slope in simulations [32]. As can be seen in figure 3, this slight decrease of the exponent significantly reduces the smallscale halo occupation and approaches the regime of alternative DM scenarios without any appreciable small-scale structures, which is commented on in $\S 4.2$.

The second row of figure 4 shows the drastic impact of $\alpha_{M}$. A minimal mass of $M_{\min }=$ $10^{-6} \mathrm{M}_{\odot}$ and $\alpha_{M}=1.85$ quadruples the signal from our reference model (solid curves in the lower panels of figure 4$), I_{l} / I_{0} \sim 4$. The signal is slightly more enhanced at energies $\lesssim 50 \mathrm{GeV}$, where absorption by the EBL is insignificant and signal from higher redshift halos

\footnotetext{
${ }^{8}$ At $z \lesssim 0.5$, we get $\varrho_{\text {halos }}=\bar{\varrho}_{\mathrm{m}, 0}$ for $\mathrm{d} n / \mathrm{d} M \propto M^{-2}$ at $M_{\min } \gtrsim 1 \mathrm{M}_{\odot}$, this number depending on the cosmology and choice of $f(\sigma)$. At higher redshifts, before the dominance of $\Omega_{\Lambda}, M_{\min }\left(\varrho_{\text {halos }}(z) \equiv \bar{\varrho}_{\mathrm{m}, 0}\right)$ quickly decreases.
} 


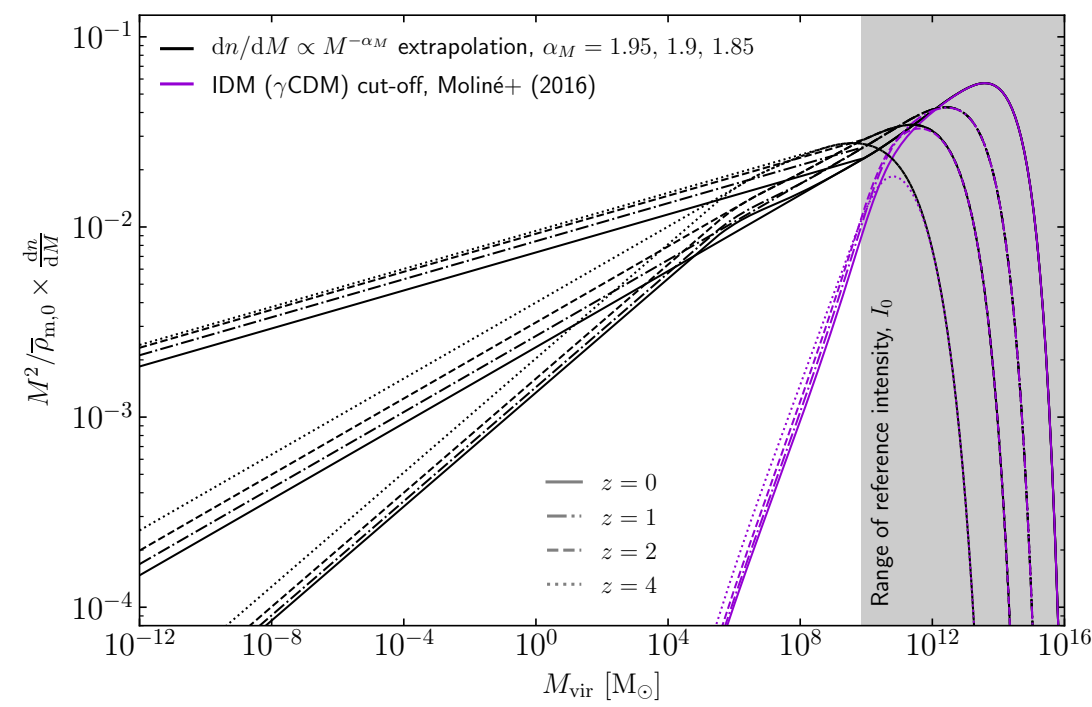

Figure 3: Halo mass function from R16 [28] with various assumptions of the low-mass scales. For the power-law extrapolations, we retain the logarithmic slope towards lower masses below $M_{\mathrm{vir}}=10^{10} \mathrm{M}_{\odot}$ from where the original mass function slope adopts the value of $\alpha_{M}$. The IDM model (purple curves) is defined by the mass function cut-off according to Eq. (11) in [24].

may contribute to the signal. Steepening $\alpha_{M}$ to our default value of 1.9 further increases the signal by additional $20 \%$ (dot-dashed curves figure 4). With $\alpha_{M}=1.95$, the increase of the signal is much larger as it is doubled compared to $\alpha_{M}=1.9$ (dashed curves, $I_{l} / I_{0} \sim 10$ ). Note, however, that combined with a small $M_{\min }$ and high low-mass halo concentrations $c_{\Delta}$, $\alpha_{M}>1.9$ may further enhance the $\gamma$-ray emission; for instance, for $\alpha_{M}=1.95$, a cut-off mass $10^{-12} \mathrm{M}_{\odot}$ would give $I_{l} / I_{0} \sim 30$.

Minimal halo mass, $M_{\min }$. In the early Universe, the kinetic decoupling of WIMPs [52, 53, 90-94] and to a lesser extent acoustic oscillations [95, 96] sets a small-scale cut-off on the mass of the smallest protohalos that can form. Numerical simulations have confirmed that such subhalos might survive until today [97]. A consistent calculation of the minimal mass associated with specific WIMP candidates was discussed in [51, 52], finding a range $\left[10^{-12}-10^{-4}\right] \mathrm{M}_{\odot}$. The third row in figure 4 shows the impact of changing this minimal mass. Following [52], we take a cut-off mass in $\left[10^{-9}-10^{-3}\right] \mathrm{M}_{\odot}$ for $m_{\chi}=100 \mathrm{GeV}$ (left panels) and $\left[10^{-12}-10^{-6}\right] \mathrm{M}_{\odot}$ for $m_{\chi}=10 \mathrm{TeV}$ (right panel). For $\alpha_{m}=1.9$, going down to smaller cut-off masses does only slightly further increase $I_{l} / I_{0}$, but would have a more drastic effect associated with a larger mass slope $\alpha_{m} \gtrsim 1.95$, as has been discussed in the previous paragraph (see figure 9 in appendix B for the flux multiplier per halo mass).

Inner profile of micro-halos. Whereas the slope of dark matter halos seems robustly determined at late stages of their evolution (see $\S 3.1$ ), recent studies have shown that halos close to the free streaming scale could be cuspier than the NFW profile, with slopes as steep as -1.5 [61, 98-102]. We calculate $I_{l}$ for extreme Einasto slopes (0.15 and 0.22), NFW, and the micro-halo model of Ishiyama (2014), see Eq. (2) in [61]. In the next-to-last row of Figure 4, we show $I_{l} / I_{0}$ in which the same profile model is used in $I_{0}$ and $I_{l}$ for consistency. The Ishiyama case which corresponds to NFW profiles for high-mass halos and steeper profiles 

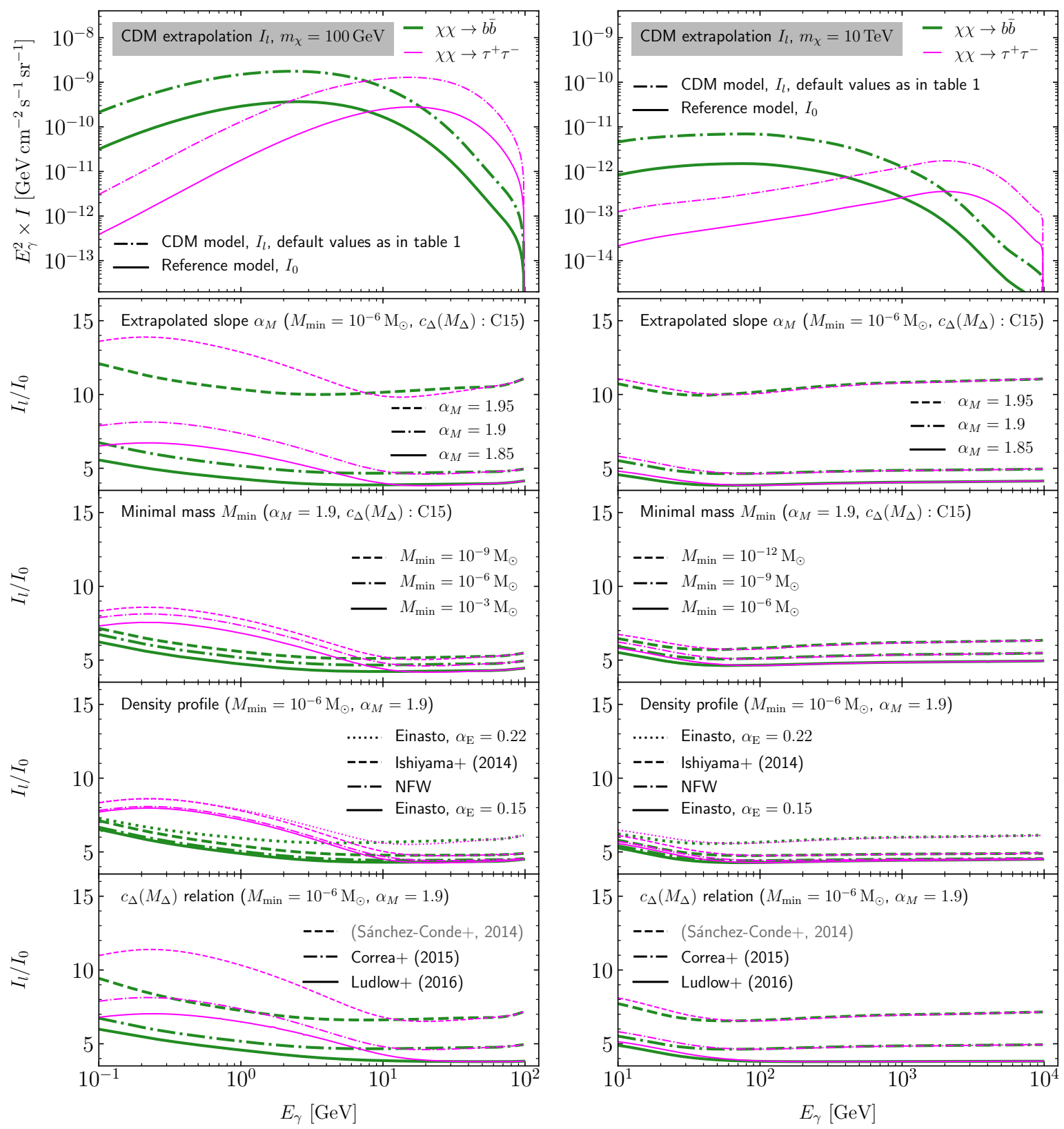

Figure 4: Top row: Intensity $I_{l}$ (all halos, no subhalos) for the same WIMP configurations as in figure 1 , where we also report $I_{0}$ for comparison purpose. Remaining rows: Ratios of the $I_{l} / I_{0}$ varying field halo properties (table 1). The bracketed model is excluded from the error budget. See discussion in $\S 3.2$.

for low-mass halos gives a $\sim 15 \%$ increase w.r.t. the NFW case. The two Einasto cases 9 encompass all the other configurations with $I_{l} / I_{0} \sim 5-9$.

Extrapolated mass-concentration-redshift parametrisation. The models of [80, 81], extrapolating $c(M, z)$ down to the lowest masses, have been widely used alternatives to

\footnotetext{
${ }^{9}$ The steeper $\alpha_{E}=0.15$ profiles give a smaller $I_{l} / I_{0}$ than $\alpha_{E}=0.22$ profiles, contrarily to the ordering seen in figure 6 . This is because both $I_{l}$ and $I_{0}$ are changed here.
} 
estimate annihilation signals for charged and neutral particles [e.g. 8, 40, 103]. In recent years, refined semi-analytical models were able to reproduce a wide range of simulations in different cosmologies [29, 30]. We use the ready-to-use parametrisations App. B1 of [29] and App. C of [75] appropriate for a Planck cosmology. We refer the reader to these papers and references therein for systematic comparisons with previous $c(M, z)$ relations. In the last row of Figure 4, we use C15 [29] as default and compare to L16 [75], but also to S14 [33] to study the impact of having a different redshift dependence. We find that L16 is $\sim 20 \%$ below C16, while S14 is $\sim 40 \%$ above. There is no simple explanation of these differences as they result from the interplay of crossing concentrations at different masses and redshifts for the various models (see figure 8 in appendix B).

\subsubsection{Boost from DM halo substructures}

In the hierarchical structure formation, host halos contain a population of subhalos with sub-subhalos, etc. down to the smallest scale. The annihilation signal from each individual halo is boosted from these populations. The boost increases with the mass [33], with only mild boosts $\lesssim 1.5$ for small halos and boost values up to 10 [40] or 100 [63] for galaxy clusters. In CLUMPY, the boost can be calculated for any distribution of subhalos down to any level of sub-substructures. We only consider the first level in the results below, as next levels only marginally contribute to the overall signal (e.g., Figure 1 of [35]). The boost depends on the subhalo properties, i.e. their number, their mass and spatial distributions, and their DM profiles. Dedicated numerical simulations of the subhalo population have been performed for Milky-Way like galaxies [62, 104-106] and galaxy clusters [28, 63, 107], but they cannot explore subhalo masses below $M \sim 10^{5} \mathrm{M}_{\odot}$. Hence, for the boost calculation, low-mass subhalo properties must also be extrapolated down to the minimum subhalo mass used in the calculation. We refer the reader to [41] for a thorough discussion of the subhalo properties and likely range of their parameters in the context of dark clumps detection in the Milky-Way at $z=0$. Here, we need in addition to consider the redshift dependence and host halo mass dependence.

As in the previous sections, we discuss in turn the various ingredients and their impact on the overall boost. To start with, the top panel of figure 5 shows, for the reference subhalo configuration (see table 1 ), the intensity accounting for the boost, $I_{\mathrm{b}}$, compared to the noboost case $I_{l}$. The boost is not even a factor two, which is understood as follows. First, the overall boost is the integration of the individual boosts of all host halos over redshifts and masses. As illustrated in appendix B and figure 9, the trend is to have a decreasing boost with decreasing $M_{\text {host }}$ and increasing $z$. At $z=0$, the boosts we obtain are consistent with the results of [33]; the decrease with $z$ is related to the decreasing concentration of halos with redshift. Second, as seen in the previous section, the intensity from field halos in the mass range below $10^{10} \mathrm{M}_{\odot}$ dominates the high-mass range contribution ( $I_{l}$ compared to $I_{0}$ in the top panel). In this mass range, mild to no boost is expected, so that this combines to give an overall very mild boost. In more details, the subhalos parameters play as follows.

Mass fraction $f_{\text {subs }}$ in subhalos. Studying the mass and redshift evolution of $f_{\text {subs }}$ from several numerical simulations, [108] found that $f_{\text {subs }}$ grows from $6 \%$ for $10^{10} \mathrm{M}_{\odot}$ halos to $17 \%$ for $10^{15} \mathrm{M}_{\odot}$ halos, and that $f_{\text {subs }}$ increases for more recently formed halos of a given mass. This range may be even larger, with a value of $2 \%$ obtained for galaxy halos in [62] and $30 \%$ for galaxy cluster halos in [63]. In their DGRB calculation, the Fermi-LAT collaboration even argue for a value $f_{\text {subs }}=0.45$ [13]. To encompass the range of possible values for the 
full range of halo masses and redshifts, we vary $f_{\text {subs }}$ from 0.1 to 0.4 ( 0.2 is our default value) in the second panel of figure 5 . This leads to a variation of the ratio $I_{\mathrm{b}} / I_{l}$ in $\sim 1.2-2$; smaller fractions would converge to no boost.

Slope $\alpha_{m}$ of subhalo mass function. We parametrise the subhalo mass function as a pure power-law, $\mathrm{d} n / \mathrm{d} m \propto m^{-\alpha_{m}}$, between $m_{\min }$ and $m_{\max }=10^{-2} \times M_{\text {host }}$ (using $10^{-1} \times$ $M_{\text {host }}$ lowers the result by $5 \%$ only). The slope $\alpha_{m}$ in numerical simulations is typically found in $1.85 \lesssim \alpha_{m} \lesssim 1.95$ [62]. Note that the slope reported in some recent simulations indicates $\alpha_{m} \sim 1.75$ [28, 107], but this assumes a power-law with an exponential cut-off, which translates into $\alpha_{m} \sim 1.9$ for a pure power law. A recent meta-analysis of the subhalo population from many simulations finds a good agreement between all the simulations, with $\alpha_{m} \sim 1.85$ [109] despite the use of different halo finders. In the third panel of figure 5, we vary this slope from 1.85 to 1.95 (with 1.9 the default value) and find that this has almost no impact on the result.

Minimal subhalo mass, $m_{\min }$. Assuming that all subhalos from the free streaming scale survive in their host halos, we match the minimal mass of the subhalos to that of the minimal mass taken for field halos (see $\S 3.2$ ). The fourth row of figure 5 shows a small scatter of $\lesssim 15 \%$ between the various mass cut-offs assumed. This indicates that unless all subhalos are destroyed up to masses much larger than $m_{\min }=10^{-3} \mathrm{M}_{\odot}$, the overall boost of the extragalactic signal is not very sensitive to the exact minimal mass of the subhalos.

Density profiles of subhalos. As for field halos, we test the impact of various density profiles for subhalos, including two extreme values for the Einasto slope, using an NFW profile, or the Ishiyama model with cuspier profiles for micro-halos. The result on the boost is shown in the next-to-last row of figure 5 , with $I_{\mathrm{b}} / I_{0}$ varying in $\sim 1.4-1.7$.

Mass-concentration-redshift relation (and subhalo spatial distribution). Microhalos can be tidally disrupted or partially stripped preferentially in the inner parts of galaxies $[106,110,111]$. This impacts the concentration of subhalos compared to field halos [112, 113], making surviving subhalos more concentrated in the inner parts of the halos. While this has an important effect for the annihilation signal in the Galaxy and dark clump detection (e.g., [41]), this mostly affects the inner parts which do not contribute much to the total annihilation signal. Moreover, beside the modelling of [113] for Galactic subhalos at $z=0$, a complete study of the subhalo mass-concentration relation with mass and redshift is lacking. For this reason, we assume the same concentration for field and subhalos, and the last row of figure 5 shows a scatter of $\pm 15 \%$ from the different models used. For the sake of completeness, we also study the impact of the subhalo spatial distribution: antibiased distributions have been obtained in DM simulations of galaxies [62] and galaxy clusters [63]. Using an unbiased distribution, i.e., the same profile as for the host, or the two above biased distributions have a marginal effect $(<1 \%)$ on $I_{\mathrm{b}} / I_{l}$.

\section{Discussion}

The exotic $\gamma$-ray intensity from DM annihilation has been previously computed by various authors and we provide comparisons to some of these earlier studies in figure 6. Our results are given by the green lines as follows: (i) the 'robust' lower bound $I_{0}$ described in $\S 3.1$ is plotted in solid green lines, (ii) the calculation extrapolated to the minimum field halo mass $I_{l}$ $(\S 3.2 .1)$ is given by the green dashed lines and (iii) finally, the intensity estimation including 

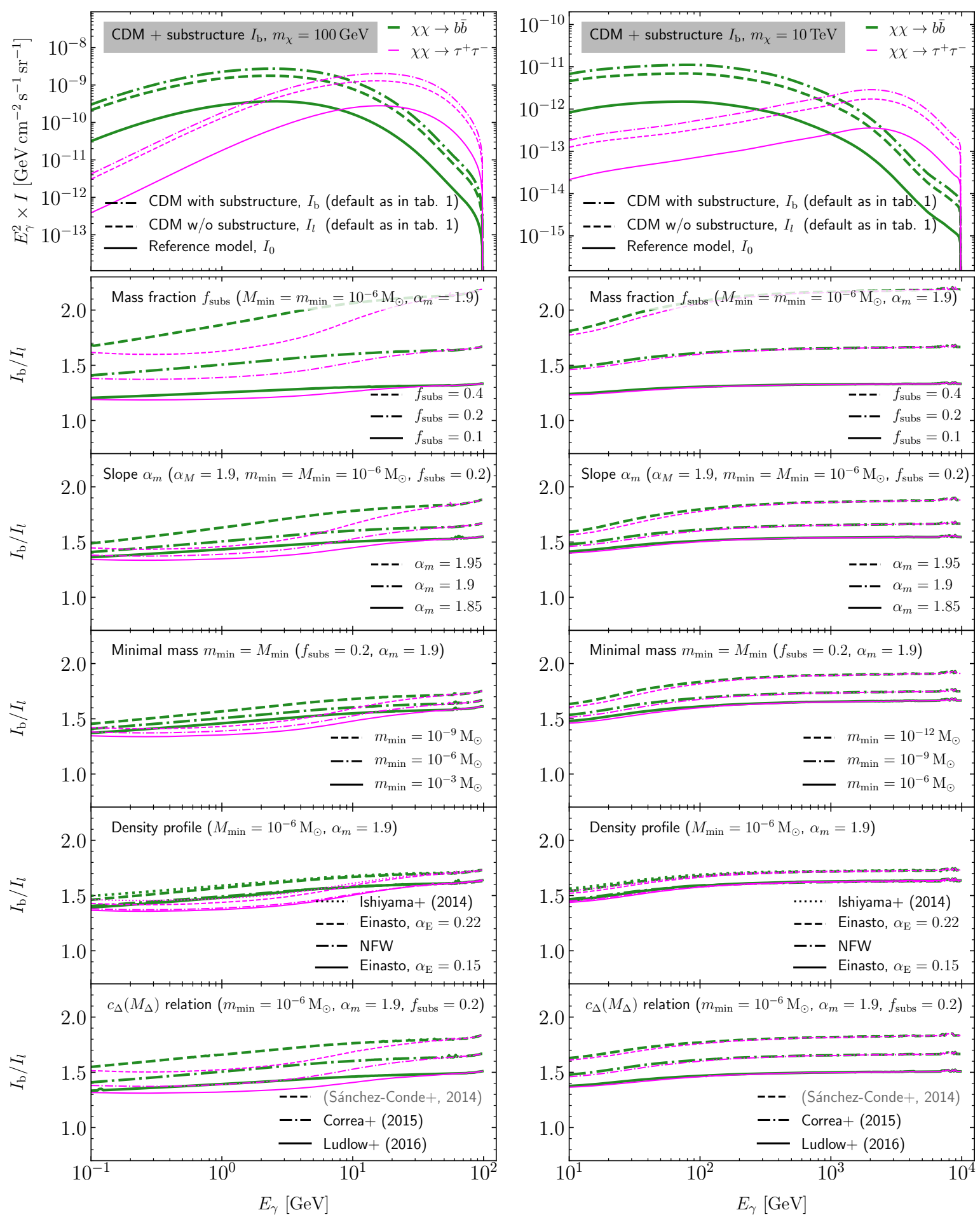

Figure 5: Top row: Intensity $I_{\mathrm{b}}$ (all halos with subhalos) for the same WIMP configurations as in figure 1 , where we also report $I_{0}\left(M \geq 10^{10} \mathrm{M}_{\odot}\right.$, no subhalos) and $I_{l}$ (all halos, no subhalos) for comparison purpose. Remaining rows: Ratios of $I_{\mathrm{b}} / I_{l}$ varying subhalo properties (table 1 ). The bracketed model is excluded from the error budget. See discussion in $\S 3.2 .2$. 
boost from subhalos $I_{\mathrm{b}}(\S 3.2 .2)$ is shown as dotted-dashed lines. The green-shaded bands correspond to the cumulative uncertainties summarised in table 1 on the lower bound from high-mass halos only, $I_{0}$, and the CDM cases including small-scale structure. The diffuse $\gamma$-ray background measured by Fermi-LAT [114] and the systematic uncertainty band from the foreground modelling are given in blue.

\subsection{Comparison to other works}

Except when comparing to the recent estimation of Moliné et al. (2016) [24] (lower panel, violet), the estimated $\gamma$-ray intensity derived in this work is lower than the previously published results of Ullio et al. (2002, [8], black solid lines), Ando et al. (2013, [45], orange solid line) and Ackermann et al. (2015, [13], red solid line). Among the many studies led on the topic, we selected these four analyses for comparison as, as a whole, they are representative of the evolution of the exotic extragalactic $\gamma$-ray emission calculations in the last fifteen years. The origins of the differences with our estimation depend on the study under scrutiny:

- In [8], the authors used a Moore parametrisation for the DM halo profiles and a mass concentration according to Bullock et al. (2001, [80]), which will both increase the intensity (black solid line in top and middle panels). The Moore DM profile, with an inner logarithmic slope of 1.5, diverges at small radii and can yield very large values of the luminosity in Eq. (2.6).

- While somewhat lower than that of [8], the prediction of [45] (orange line in top panel) is still much higher than our estimation. This is understood as they used an effective subhalo boost model fitted to the results of [63], typically $\sim 20$ at halo masses of $M=10^{10} \mathrm{M}_{\odot}, \sim 100$ at $10^{12} \mathrm{M}_{\odot}$, and $\sim 1500$ at $10^{15} \mathrm{M}_{\odot}$. Adopting such values would indeed bring the total intensity $I_{\mathrm{b}}$ to the level of their result. Since then, such large boosts have been excluded by several authors [33, 40, 113], including this work (see figure 9), when using physically-motivated mass-concentration relations at low masses.

- We also compare our result to that of the Fermi-LAT collaboration [13] (red curve in middle panel), whose estimate is consistent with the upper limit of our uncertainty band. Conversely to the previous cases, the difference with our reference is not due to a single major feature of their modelling, but from small differences in the extrapolation to lower mass (e.g., steeper slope of the subhalo mass distribution, and possibly of the mass function extrapolation, larger subhalo mass fraction) which yield, when combined, the factor $\sim 5$ between $I_{\mathrm{b}}$ and their result in the middle panel of figure 6 . The authors also explored the uncertainties using the non-linear power spectrum instead of the halo model approach we used, noting that the many dependencies of the halo model render this task more difficult. It is interesting to note that the uncertainty band we find by varying all the various ingredients in the halo model is similar, although twice smaller, to what [13] find using the power spectrum approach.

\subsection{A word on IDM (SIDM $/ \gamma$ CDM) models}

So far, we have remained in the framework of collisionless, cold DM. Beyond this paradigm, the concept of interacting DM (IDM) has raised the attention of the community in the last years as a possible solution to various observational tensions on subgalactic scales between collisionless CDM and observations. These include the observation of pronounced DM halo

cores from dwarf galaxy to cluster sizes $[115,116]$, the problems of the diversity of rotation 


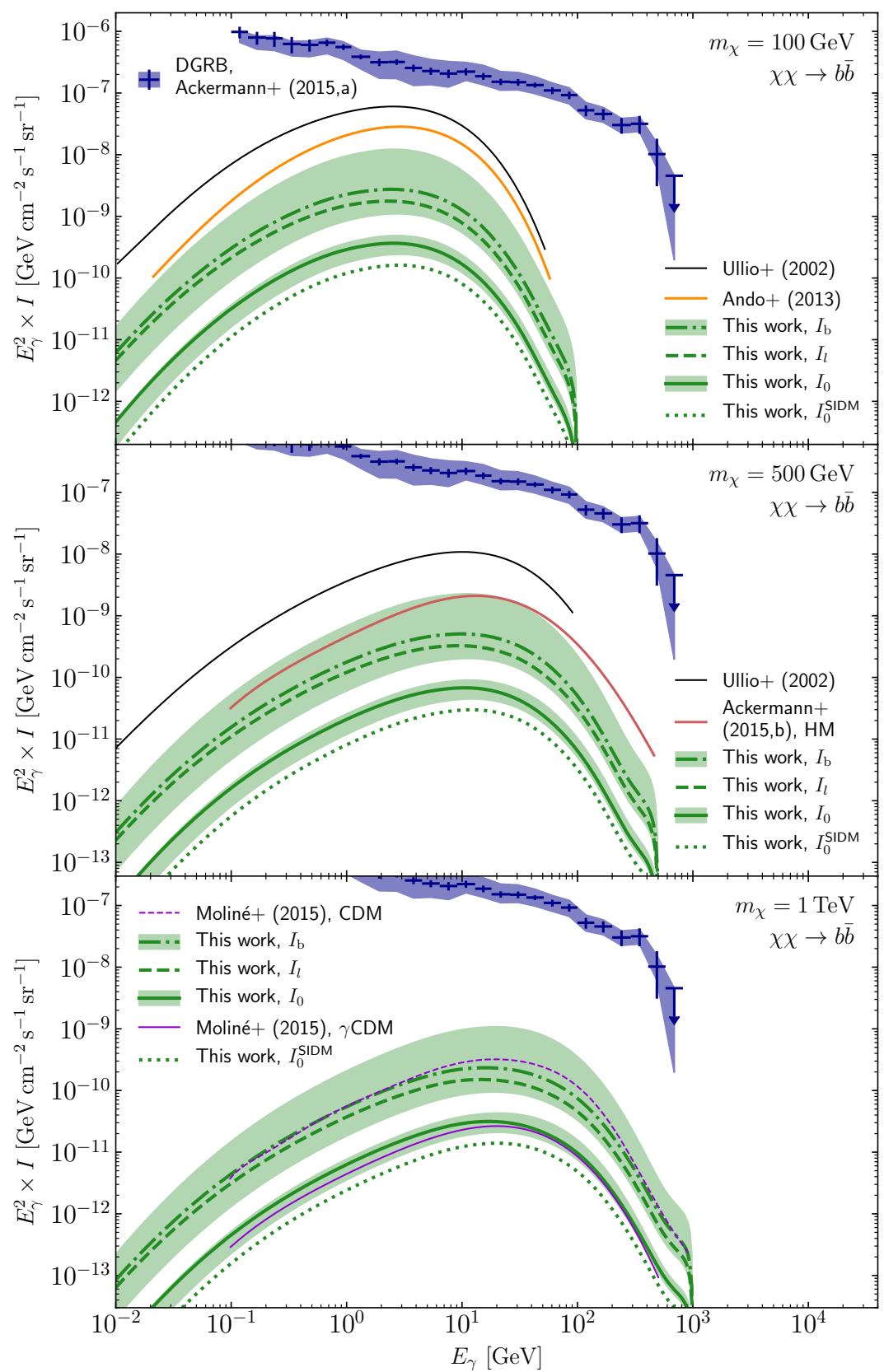

Figure 6: Extragalactic DM annihilation intensity for $m_{\chi}=100$ (top), 500 (middle), and $10^{3} \mathrm{GeV}$, for pure $\chi \chi \rightarrow b \bar{b}$ annihilation and $\langle\sigma v\rangle=3 \times 10^{-26} \mathrm{~cm}^{3} \mathrm{~s}^{-1}$ : our results for collisionless CDM are shown for high-mass halos only $\left(M \geq 10^{10} \mathrm{M}_{\odot}\right.$, solid green curves), the intensity of all halos without (dashed green curves) or with (dot-dashed green curves) substructures. The green uncertainty bands are obtained from extreme choices of the ingredients in table 1. The total intensity is compared to selected previous works (black [8], orange [45], red [13], and violet [24] curves). The intensity for the SIDM scenario, as discussed in $\S 4.2$, is shown as a dotted green curve. We also report the DGRB intensity as measured by Fermi-LAT [114] (foreground model A), where the blue shaded band denotes their systematic uncertainty due to the Galactic foreground modelling. 
curves [117] and missing satellites [118], and the "too-big-to-fail" problem [119]. In contrast to warm DM (WDM) made of sub-MeV particles as a possible solution to CDM small-scale issues, IDM may solve these problems while at the same time preserving the remarkable successes of CDM. Most importantly in our context and in contrast to WDM, IDM could still consist of cold and heavy DM particles annihilating into high-energy $\gamma$-rays.

In a self-interacting DM (SIDM) scenario, the DM particle has a non-negligible cross section, $\sigma_{\mathrm{el}}$, of mutual weak elastic scatterings [120]. A scattering cross section $\sigma_{\mathrm{el}}$ in the order of magnitude of $\sigma_{\mathrm{el}} / m_{\chi} \sim 0.1 \mathrm{~cm}^{2} / \mathrm{g}$ would be still in agreement with observation, e.g., the non-observation of self-interactions in cluster mergers [121] or halo-shape constraints [122] (see also [120] for further references). At the same time, such a self-interaction would be large enough to repeal the diversity and cusp-vs-core problems by increasing the scatter in concentrations and thermalising the inner halos, removing the inner density cusps of Einasto or NFW halos. On the other hand, it has been shown that a velocity-independent $\sigma_{\mathrm{el}} / m_{\chi} \sim$ $0.1 \mathrm{~cm}^{2} / \mathrm{g}$ is too low to solve the missing-mass problem (i.e., not able to significantly reduce the number density of small-mass halos) [123]. Therefore, elastic scattering between the DM and photons ( $\gamma \mathrm{CDM}$ ") or neutrinos has been proposed as a more general class of interacting DM (IDM) [124]. It has been shown that $\gamma$ CDM scatterings with cross sections on the electroweak scale likewise reduce the core concentrations of MW-like DM halos and can also efficiently suppress structures at the dwarf-galaxy scale, $M \lesssim 10^{10} \mathrm{M}_{\odot}[24,125]$. As shown in figure 4, [24] find, for a $\gamma \mathrm{CDM}$ scattering with $\sigma_{\mathrm{el}} / m_{\chi}=7.5 \times 10^{-10} \mathrm{~cm}^{2} / \mathrm{g} \sim 1 \mathrm{nb} / \mathrm{GeV},{ }^{10}$ the halo mass function is suppressed at just around the scale of our reference model, $I_{0}$, with a half-mode mass of $M_{\mathrm{hm}}=4.3 \times 10^{9} \mathrm{~h}^{-1} \mathrm{M}_{\odot}$.

We shortly explore the impact of thermalised halo cores in such models, as such a flattening of the central densities may significantly affect the $\gamma$-ray signal from DM annihilation. To this purpose, we remain in the framework of SIDM and assume a SIDM scattering cross section of $\sigma_{\mathrm{el}} / m_{\chi} \sim 1 \mathrm{~cm}^{2} / \mathrm{g}$. Although in tension with several constraints, this allows to conservatively estimate a maximal reduction of the $\gamma$-ray intensity from cored inner DM halos. For such a SIDM cross section, the authors of [126] provide a simple scaling relation, to which we add a redshift evolution of the comoving core density according to

$$
\rho_{\text {core }}\left(M_{\text {vir }}, z\right)=\frac{0.029}{(1+z)^{3}} \frac{\mathrm{M}_{\odot}}{\mathrm{pc}^{3}} \times\left(\frac{M_{\text {vir }}}{10^{10} \mathrm{M}_{\odot}}\right)^{-0.19}
$$

for a Burkert halo profile [127]

$$
\rho^{\text {Burkert }}(r)=\frac{\rho_{\text {core }} r_{\text {core }}^{3}}{\left(r+r_{\text {core }}\right)\left(r^{2}+r_{\text {core }}^{2}\right)} .
$$

This relation corresponds to core radii of $r_{\text {core }}\left(10^{10} \mathrm{M}_{\odot}\right)=2.22 \mathrm{kpc}$ and $r_{\text {core }}\left(10^{15} \mathrm{M}_{\odot}\right)=$ $244 \mathrm{kpc}$ at $z=0$, respectively. At higher redshifts, Eq. (4.1) results in halo concentrations $c_{\mathrm{vir}}(z) \sim c_{\mathrm{vir}}(0) /(1+z)$ which we have found to provide lower intensities compared to more recent prescriptions (see $\S 3.1$ ). We retain $M_{\min }=10^{10} \mathrm{M}_{\odot}$ for this assessment of DM selfinteractions, although smaller structure may be still present for SIDM. We obtain that a core condition according to Eq. (4.1) reduces the halo luminosities by approximately a factor 2 in the range $10^{10} \mathrm{M}_{\odot} \lesssim M \lesssim 10^{15} \mathrm{M}_{\odot}$ at $z=0$ and more at higher redshifts. As shown in figure 6 (green dotted curves), the intensity $I_{0}^{\text {SIDM }}$ from halos with SIDM cores is a factor of $2-5$ (depending on $E_{\gamma}$ ) lower than the corresponding reference intensity, $I_{0}$.

\footnotetext{
${ }^{10}$ See $[124]$ for a detailed motivation of the chosen value.
} 


\section{Conclusions}

In this work, we have reassessed the isotropic emission of $\gamma$-rays for extragalactic annihilations of WIMPs in a clumpy $\Lambda$ CDM Universe. We have also ranked the various sources of uncertainties, in order to identify which ingredients dominate the error budget. The results are based on the latest available knowledge about structure formation in a Planck cosmology.

For CDM, we first calculate the intensity $I_{0}$ from DM halos with $M \geq 10^{10} \mathrm{M}_{\odot}$, a mass range where no extrapolation is required. This contribution constitutes a lower bound on the $\gamma$-ray emission from extragalactic DM and we find $I_{0}$ can be robustly estimated within a factor 2. Accounting for the population of halos and subhalos down to the smallest masses, the total intensity, $I_{\mathrm{b}}$, is a factor $\sim 10$ larger than $I_{0}$ and bracketed by a one order of magnitude uncertainty band. Recent estimations of $I_{\mathrm{b}}$ (including our own) tend to find lower values than earlier studies; this trend can be traced to differences in the mass function extrapolation and to the recent predictions of smaller mass concentrations in small-scale halos. In particular, we conclude in a marginal boost $(\sim 1.5)$ of the signal by halo substructures.

The fiducial spectrum of the intensity $I_{\mathrm{b}}$ obtained in this work is a factor 5 smaller (with slightly smaller uncertainties) than that derived in the Fermi-LAT analysis [13]. Taken at face value, this would relax the corresponding DM exclusion limit by the same amount, making the DGRB an even less competitive target w.r.t. dwarf spheroidal galaxies.

For SIDM with $\sigma_{\mathrm{el}} / m_{\chi} \sim 1 \mathrm{~cm}^{2} / \mathrm{g}$, we find that the signal of our lower bound $I_{0}$ is further reduced by a factor $\sim 3$ compared to the collisionless case. As we generally expect fewer or even no boost from small structures in interacting DM scenarios, the extragalactic signal may remain three (for $m_{\chi}=100 \mathrm{GeV}$ ) to four (for $m_{\chi}=1 \mathrm{TeV}$ ) orders of magnitudes below the measured DGRB intensity for a canonical annihilation cross section of $\langle\sigma v\rangle=$ $3 \times 10^{-26} \mathrm{~cm}^{3} \mathrm{~s}^{-1}$. This indicates that if a $\gamma$-ray signal from DM self-annihilations is seen in dwarf spheroidal galaxies, whose signal is not expected to change much between interacting and collisionless DM scenarios, a non-observation of a corresponding signal in the DGRB could be used to probe properties of elastic DM interactions in SIDM and $\gamma \mathrm{CDM} / \nu \mathrm{CDM}$ models.

All the results presented in this work have been performed with a soon to be released version of the CLUMPY code. Using the latter, the calculation could be easily repeated to assess neutrino signals from extragalactic DM, for which the various final state neutrino spectra are already included in the code.

\section{Acknowledgments}

We thank J. Lesgourgues for helpful input on the CLASS power spectrum. This work has been supported by the Research Training Group 1504, "Mass, Spectrum, Symmetry", of the German Research Foundation (DFG), by the "Investissements d'avenir, Labex ENIGMASS", and by the French ANR, Project DMAstro-LHC, ANR-12-BS05-0006.

\section{A Mass conversion between different $\Delta$ definitions}

Let us assume a generic halo density profile, $\rho_{\text {halo }}\left(r ; \rho_{-2}, r_{-2}, \vec{\alpha}\right)$ with a given set of $\vec{\alpha}$ : for instance, $\vec{\alpha}=\alpha_{E}$ for an Einasto or $\vec{\alpha}=(\alpha, \beta, \gamma)=(1,3,1)$ for an NFW profile. We want to calculate its mass $M_{\Delta_{1}}$ for a corresponding $\Delta_{1}$, given a mass-concentration relation w.r.t. 
$\Delta_{\text {ref }}\left(\neq \Delta_{1}\right)$

$$
c_{\Delta_{\text {ref }}}\left(M_{\Delta_{\text {ref }}}\right)=\frac{R_{\Delta_{\text {ref }}}}{r_{-2}}\left(M_{\Delta_{\text {ref }}}\right) .
$$

Provided a density profile of the generic form

$$
\rho_{\text {halo }}=\rho_{-2} \times \widetilde{\rho}\left(r / r_{-2} ; \vec{\alpha}\right)=\rho_{-2} \times \widetilde{\rho}(x ; \vec{\alpha}),
$$

one can solve the implicit equation

$$
\frac{M_{\Delta_{i}}}{M_{\Delta_{\text {ref }}}} \times \frac{\int_{0}^{c_{\text {ref }}} x^{2} \widetilde{\rho}(x ; \vec{\alpha}) \mathrm{d} x}{\int_{0}^{c_{\text {ref }}^{\prime}} x^{2} \widetilde{\rho}(x ; \vec{\alpha}) \mathrm{d} x}=1
$$

for $M_{\Delta_{\text {ref }}}$, given $\Delta_{i}=\Delta_{1}$, and with the expressions

$$
\begin{aligned}
& c_{\mathrm{ref}}:=c_{\Delta_{\mathrm{ref}}}\left(M_{\Delta_{\mathrm{ref}}}, z\right), \\
& c_{\mathrm{ref}}^{\prime}:=c_{\Delta_{\mathrm{ref}}}\left(M_{\Delta_{\mathrm{ref}}}, z\right) \times\left(\frac{M_{\Delta_{i}}}{M_{\Delta_{\mathrm{ref}}}} \times \frac{\Delta_{\mathrm{ref}}(z)}{\Delta_{i}(z)}\right)^{\frac{1}{3}} .
\end{aligned}
$$

Now, knowing $M_{\Delta_{\text {ref }}}$, Eq. (A.3) can be solved a second time for $\Delta_{i}=\Delta_{2}$ to directly convert the halo mass from an arbitrary $\Delta_{1}$ to $\Delta_{2}$ without the need to determine $\rho_{-2}$ and $r_{-2}$ of the halo. Although computationally more expensive, this rigorous algorithm is more flexible and precise for arbitrary choices of mass-concentration relations and halo profiles than the approximate translation recipe from [128] which we have used in an earlier work [41].

\section{B Behaviour of the various terms in the intensity multiplier}

In this appendix, we provide more details about the ingredients we use to compute the intensity multiplier, Eq. (2.2), and intermediate results.

Halo mass function/multiplicity function. In this work, we have used various descriptions of the multiplicity function, $f(\sigma, z)$ (Eq. (2.3)), in different cosmologies. For validation purpose of the discussion in $\S 3.1$, we show in figure 7 the underlying halo mass functions rescaled to $\Delta=\Delta_{\text {vir }}$ : this emphasises the impact of changing the multiplicity function in a given cosmology (right panel of figure 7) or changing the cosmology underlying the linear matter power spectrum to compute $\sigma(M)$, Eq. (2.4), (left panel). Note the remarkable concordance of the mass functions from T08 [32] and B16 [55] in their "original" cosmologies and in the $\Delta_{\text {vir }}$ prescription (blue and green curves in the central panel).

Mass-concentration-redshift parametrisation $c(M, z)$. Figure 8 displays the different models of halo concentrations compared in this work for an overdensity definition of $\Delta_{\text {vir }}$ according to Eq. (3.3). The original concentration relations for all these three models are given w.r.t $\Delta_{c}=200$. In this figure, we used the algorithm described in the previous appendix A to translate the concentration relations to different choices for $\Delta$, assuming an Einasto halo density profile with $\alpha_{\mathrm{E}}=0.17$. This rescaling between different definitions for $\Delta$ explains why the green curves for the model S14 are not overlapping when displaying $(1+z) c_{\mathrm{vir}}$ : While the original prescription from [33] gives $c_{200, \mathrm{c}}\left(M_{200, \mathrm{c}}, z\right)=c_{200, \mathrm{c}}\left(M_{200, \mathrm{c}}, z=0\right) /(1+z)$, our 


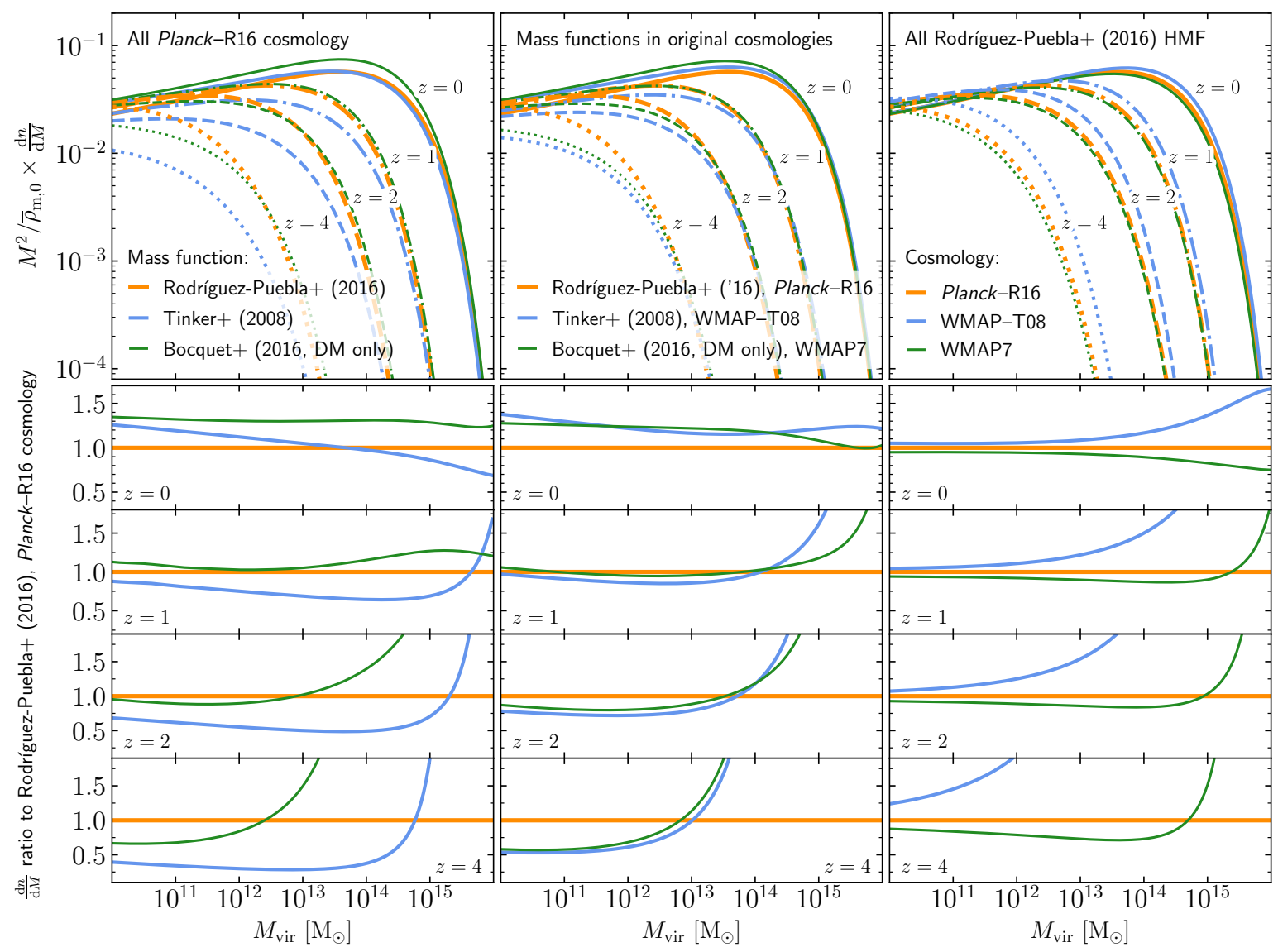

Figure 7: Left panels: Comparison of different mass functions in the same cosmology of Planck-R16 [28]. Central panels: Comparison of different mass functions in the cosmology of the simulations they were derived from. Right panels: Comparison of the mass function from [28] rescaled to different cosmologies. Note that $\bar{\varrho}_{\mathrm{m}, 0}$ differs between the cosmologies, while the ratios are given with respect to $\mathrm{d} n / \mathrm{d} M$.

recipe results in a $c_{\text {vir }}\left(M_{\text {vir }}, z\right)$ decreasing even stronger with redshift. ${ }^{11}$ In turn, the model L16 [30] (blue curves) shows a weaker scaling than $\propto(1+z)^{-1}$ in both the original description and after translation to $\Delta_{\text {vir. }}$. Our default model C15 [29] (orange curves) remarkably well scales $\propto(1+z)^{-1}$ relative to $\Delta_{\text {vir }}$ over several mass decades, $10^{-6} \mathrm{M}_{\odot} \lesssim M_{\text {vir }} \lesssim 10^{8} \mathrm{M}_{\odot}$.

One-halo luminosities, $\mathcal{L}(M, z)$, and substructure boost. Figure 9 (upper left) shows the DM one-halo luminosities $\mathcal{L}$, Eq. (2.6), for our default $c_{\Delta}\left(M_{\Delta}\right)$ model C15 [29]. Displaying $(1+z)^{3} \times \mathcal{L}$, it can be seen that the higher mean density of the early Universe - and by this, a higher annihilation rate - overcompensates the less concentrated halos at earlier epochs, and the comoving luminosity increases with redshift. This finally prevents convergence of the intensity multiplier over the considered redshift range in a collisionless CDM paradigm, as illustrated in the later figure 10. The lower panel of figure 9 shows the ratio of the emission with and without substructures, commonly referred to as the substructure boost. For the concentration model from [29], the emission is only moderately boosted by substructures by

\footnotetext{
${ }^{11}$ Note that $\Delta_{\text {vir }}(z=0) \approx 100$. For $z \gtrsim 1, \Delta_{\text {vir }}(z)$ converges towards $178<\Delta_{\mathrm{c}}=200$, as $\Omega_{\mathrm{m}} \rightarrow 1$ in the matter dominated era.
} 


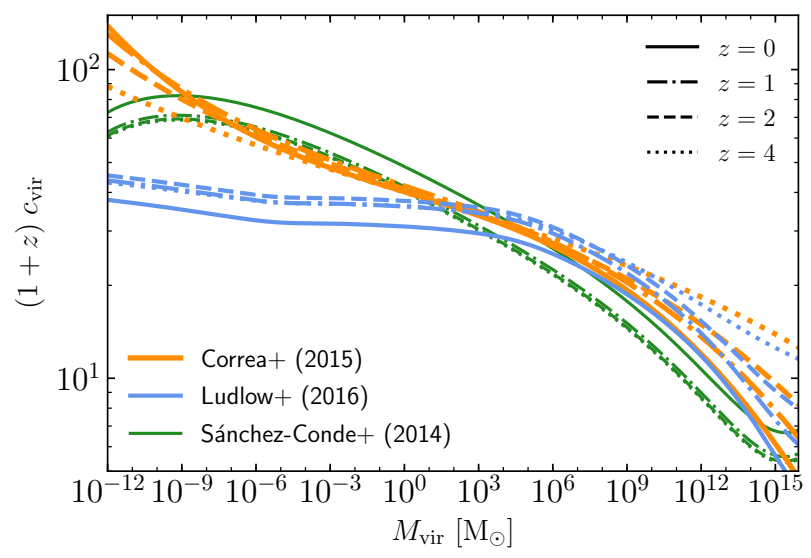

Figure 8: Mass-concentration relations considered in this work w.r.t the overdensity $\Delta_{\text {vir }}$, Eq. (3.3). The rescaling to $\Delta_{\text {vir }}$ from the original prescriptions is done according to appendix A and using an Einasto profile. Note that the model L16 [30] is based on the density variance $\sigma$, which is computed here using Eq. (2.4) and not from the analytical approximation provided in L16.
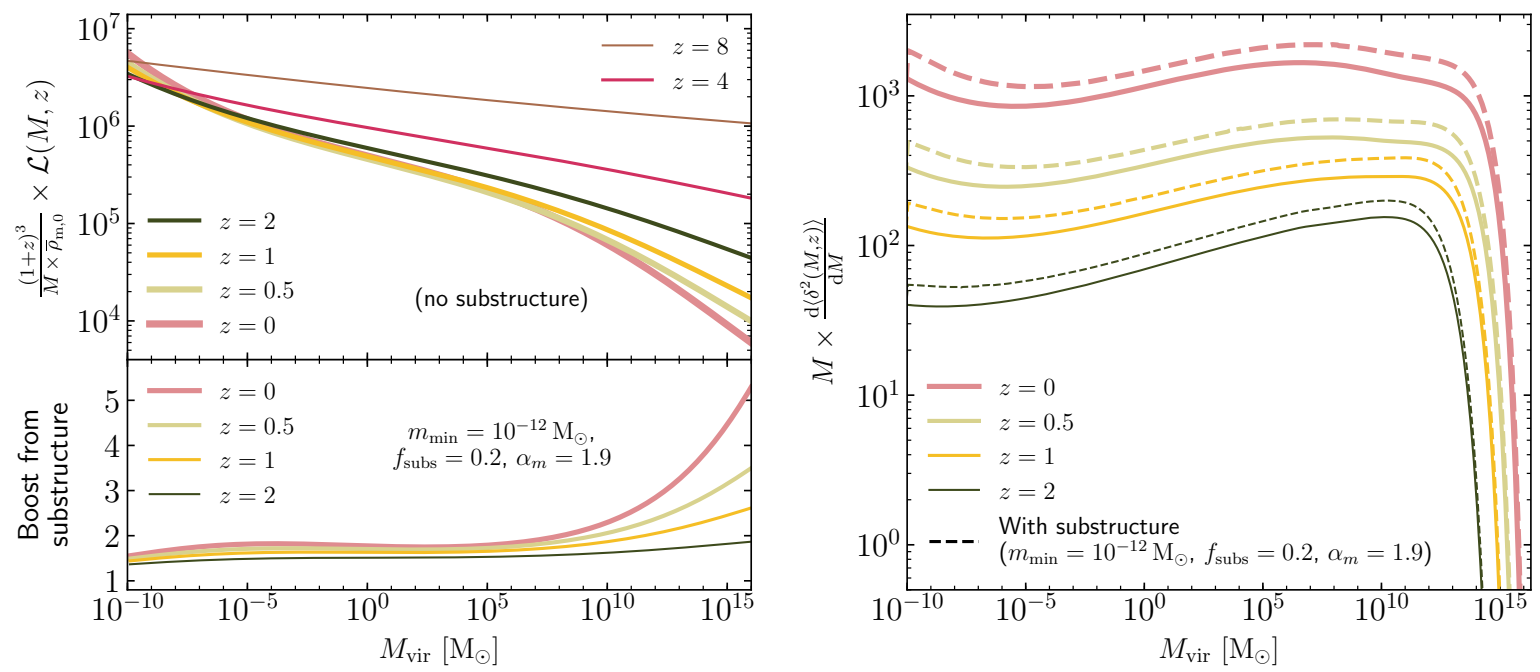

Figure 9: Contributors to the intensity multiplier, $\left\langle\delta^{2}\right\rangle$, Eq. (2.2), from different halo mass decades. Upper left panel: Comoving one-halo luminosities at different redshifts, Eq. (2.6), in the reference model and without halo substructures. Lower left panel: Boost factor to the one-halo luminosities at different redshifts due one level of halo substructures and the $c(M, z)$ model C15 [29]. It can be seen that the substructure boost decreases with redshift and host halo mass. Right panel: Integrand of the mass integral of Eq. (2.2) at $z \leq 2$ with and without substructure boost. Note that for display purpose, contrarily to the left, we do not multiply $\mathrm{d}\left\langle\delta^{2}\right\rangle / \mathrm{d} \log M$ with a factor $(1+z)^{3}$ here.

less than a factor five at galaxy cluster masses at $z=0$, and even less for smaller masses and higher redshifts.

Intensity multiplier, $\left\langle\delta^{2}\right\rangle$. In figure 9 (right) we show the contribution per mass decade to the intensity multiplier, Eq. (2.2). Here, the one-halo luminosities are multiplied with the halo number density $\mathrm{d} n / \mathrm{d} M$, which is extrapolated to the micro-halo scale according to a powerlaw extrapolation, Eq. (3.6) with $\alpha_{M}=1.9$. We show $\mathrm{d}\left\langle\delta^{2}\right\rangle / \mathrm{d} \log M$ without (solid lines) and with (dashed lines) emission boost from substructures. From this depiction it becomes 

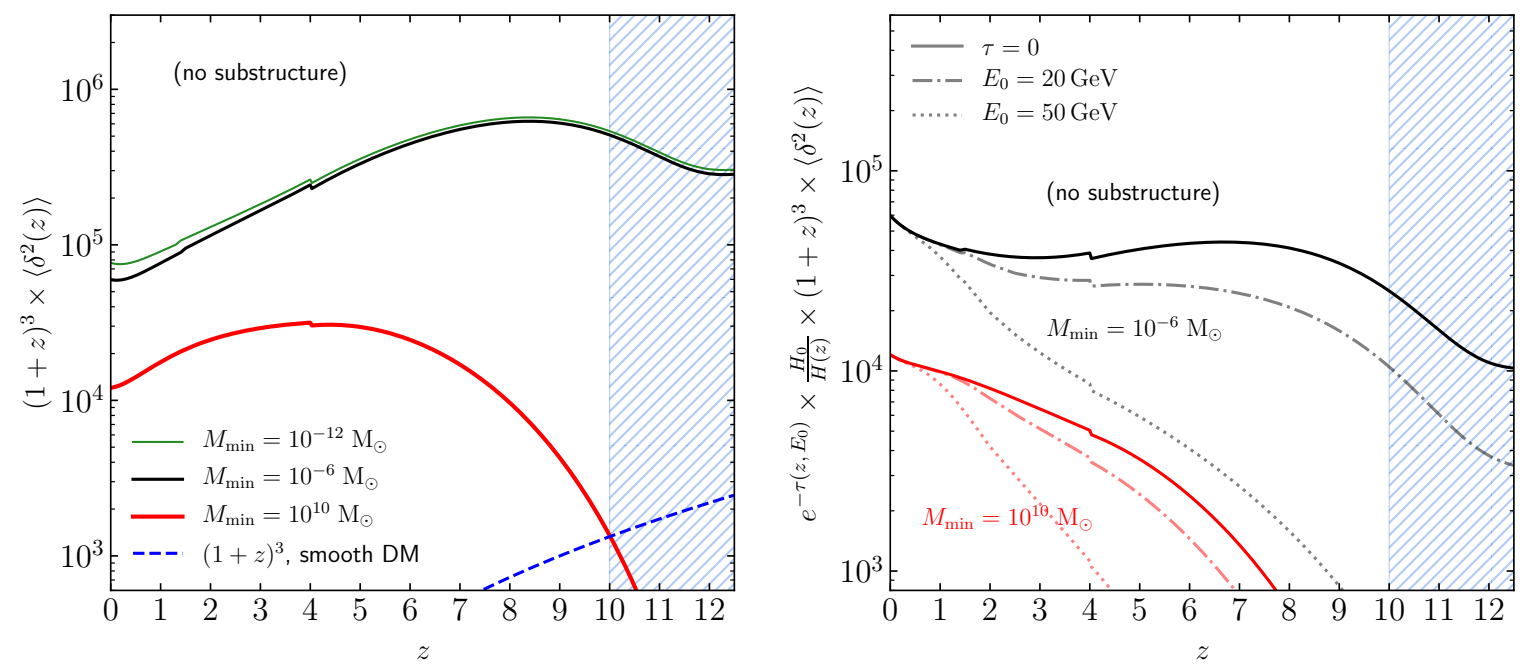

Figure 10: Redshift dependence of the intensity multiplier, $\left\langle\delta^{2}\right\rangle$, Eq. (2.2), for our reference model (red curves) and the default collisionless CDM case without substructures (black curves). On the left, we show the comoving multiplier (we additionally display the impact of adopting $M_{\min }=10^{-12} \mathrm{M}_{\odot}$, green curve), whereas on the right, the multiplier is additionally multiplied by the volume element and different EBL absorption factors. The step in the curves originates from the $c_{\Delta}$ model by [29], which connects two functional forms at $z=4$. See text for further details.

evident that a cut-off of the mass function at some minimal mass $M_{\min }$ is compulsory for the integral of Eq. (2.2) not to diverge. Previously, it has been found that for optimistic assumptions about the substructure boost, the intensity multiplier is dominated by galaxy and cluster-size halos, $M \gtrsim 10^{10} \mathrm{M}_{\odot}$ [45]. However, this dominance is not present for our more moderate assumption of the concentration model C15, as shown in figure 9 (left), for which all mass decades are similarly boosted (dashed lines).

Figure 10 finally shows the full intensity multiplier as a function of the redshift. The left panel shows that in a collisionless CDM scenario (power-law extrapolation of the mass function with $\alpha_{M}=1.9$ down to $M_{\min }=10^{-6} \mathrm{M}_{\odot}$, black curve, resp. $M_{\min }=10^{-12} \mathrm{M}_{\odot}$, green curve), the density variance remains $\left\langle\delta^{2}\right\rangle \approx \operatorname{Var}(\delta) \gtrsim 100$ until high redshifts, $z \gtrsim 10$. For our reference model, where we only consider structures and halos on comoving mass scales larger than $10^{10} \mathrm{M}_{\odot}$, the intensity multiplier from halos becomes smaller than 1 at $z \approx 10$ (red curve on the left). Note that the emission from structureless DM, $\left\langle\delta^{2}\right\rangle \equiv 1$, exceeds the emission from high-mass halos - our reference model - at redshifts $z \gtrsim 10$ (blue dashed curve on the left). On the right panel, we show the intensity multiplier multiplied with the volume element, expressed by the inverse Hubble constant, and the EBL attenuation factor. This quantity is finally integrated in Eq. (2.1) over the redshift to obtain the total extragalactic DM $\gamma$-ray intensity. However, we only integrate the intensity up to $z_{\max }=10$, and the blue hatched areas in figure 10 indicate the redshift regime which we exclude in our calculations. The contribution to the intensity from $z>z_{\max }$ is marginal when considering only masses $M \geq 10^{10} \mathrm{M}_{\odot}$ (red curves) or in the presence of EBL absorption (dotted and dashed-dotted curves), however not necessarily in the presence of small-scale clustering and no absorption (black solid line on the right). 


\section{References}

[1] M. Fornasa and M. A. Sánchez-Conde, The nature of the Diffuse Gamma-Ray Background, Phys. Rep. 598 (Oct., 2015) 1-58, [1502.02866].

[2] T. Bringmann and C. Weniger, Gamma ray signals from dark matter: Concepts, status and prospects, Physics of the Dark Universe 1 (Nov., 2012) 194-217, [1208.5481].

[3] J. E. Gunn, B. W. Lee, I. Lerche, D. N. Schramm and G. Steigman, Some astrophysical consequences of the existence of a heavy stable neutral lepton, ApJ 223 (Aug., 1978) $1015-1031$.

[4] F. W. Stecker, The cosmic $\gamma$-ray background from the annihilation of primordial stable neutral heavy leptons, ApJ 223 (Aug., 1978) 1032-1036.

[5] J. Silk and M. Srednicki, Cosmic-ray antiprotons as a probe of a photino-dominated universe, Physical Review Letters 53 (Aug., 1984) 624-627.

[6] Y.-T. Gao, F. W. Stecker and D. B. Cline, The lightest supersymmetric particle and the extragalactic gamma-ray background, A\&̈A 249 (Sept., 1991) 1-4.

[7] L. Bergström, J. Edsjö and P. Ullio, Spectral Gamma-Ray Signatures of Cosmological Dark Matter Annihilations, Phys. Rev. Lett. 87 (Dec., 2001) 251301, [astro-ph/0105048].

[8] P. Ullio, L. Bergström, J. Edsjö and C. Lacey, Cosmological dark matter annihilations into $\gamma$ rays: A closer look, Phys. Rev. D 66 (Dec., 2002) 123502, [astro-ph/0207125].

[9] M. R. Feyereisen, S. Ando and S. K. Lee, Modelling the flux distribution function of the extragalactic gamma-ray background from dark matter annihilation, J. Cosmology Astropart. Phys. 9 (Sept., 2015) 027, [1506.05118].

[10] S. Ando and E. Komatsu, Anisotropy of the cosmic gamma-ray background from dark matter annihilation, Phys. Rev. D 73 (Jan., 2006) 023521, [astro-ph/0512217].

[11] A. Cuoco, J. Brandbyge, S. Hannestad, T. Haugbølle and G. Miele, Angular signatures of annihilating dark matter in the cosmic gamma-ray background, Phys. Rev. D $7 \mathbf{7}$ (June, 2008) 123518, [0710.4136].

[12] A. A. Abdo, M. Ackermann, M. Ajello, L. Baldini, J. Ballet, G. Barbiellini et al., Constraints on cosmological dark matter annihilation from the Fermi-LAT isotropic diffuse gamma-ray measurement, J. Cosmology Astropart. Phys. 4 (Apr., 2010) 014, [1002.4415].

[13] The Fermi LAT Collaboration, Limits on dark matter annihilation signals from the Fermi LAT 4-year measurement of the isotropic gamma-ray background, J. Cosmology Astropart. Phys. 9 (Sept., 2015) 008, [1501.05464].

[14] M. Ajello, A. Albert, W. B. Atwood, G. Barbiellini, D. Bastieri, K. Bechtol et al., Fermi-LAT Observations of High-Energy Gamma-Ray Emission toward the Galactic Center, ApJ 819 (Mar., 2016) 44, [1511.02938].

[15] M. Fornasa, A. Cuoco, J. Zavala, J. M. Gaskins, M. A. Sánchez-Conde, G. Gomez-Vargas et al., Angular power spectrum of the diffuse gamma-ray emission as measured by the Fermi Large Area Telescope and constraints on its dark matter interpretation, Phys. Rev. D 94 (Dec., 2016) 123005, [1608.07289].

[16] W. Liu, X.-J. Bi, S.-J. Lin and P.-F. Yin, Constraints on dark matter annihilation and decay from the isotropic gamma-ray background, Chinese Physics C 41 (Apr., 2017) 045104, [1602.01012].

[17] M. Ackermann, A. Albert, B. Anderson, W. B. Atwood, L. Baldini, G. Barbiellini et al., Searching for Dark Matter Annihilation from Milky Way Dwarf Spheroidal Galaxies with Six Years of Fermi Large Area Telescope Data, Phys. Rev. Lett. 115 (Dec., 2015) 231301, [1503.02641]. 
[18] A. Drlica-Wagner, A. Albert, K. Bechtol, M. Wood, L. Strigari, M. Sánchez-Conde et al., Search for Gamma-Ray Emission from DES Dwarf Spheroidal Galaxy Candidates with Fermi-LAT Data, ApJ 809 (Aug., 2015) L4, [1503.02632].

[19] J. E. Taylor and J. Silk, The clumpiness of cold dark matter: implications for the annihilation signal, MNRAS 339 (Feb., 2003) 505-514, [astro-ph/0207299].

[20] K. Ahn and E. Komatsu, Cosmological lower bound on dark matter masses from the soft gamma-ray background, Phys. Rev. D 71 (Jan., 2005) 021303, [astro-ph/0412630].

[21] J. Zavala, V. Springel and M. Boylan-Kolchin, Extragalactic gamma-ray background radiation from dark matter annihilation, MNRAS 405 (June, 2010) 593-612, [0908.2428].

[22] K. J. Mack, Known unknowns of dark matter annihilation over cosmic time, MNRAS 439 (Apr., 2014) 2728-2735, [1309.7783].

[23] K. C. Y. Ng, R. Laha, S. Campbell, S. Horiuchi, B. Dasgupta, K. Murase et al., Resolving small-scale dark matter structures using multisource indirect detection, Phys. Rev. D $\mathbf{8 9}$ (Apr., 2014) 083001, [1310.1915].

[24] Á. Moliné, J. A. Schewtschenko, S. Palomares-Ruiz, C. Bøhm and C. M. Baugh, Isotropic extragalactic flux from dark matter annihilations: lessons from interacting dark matter scenarios, J. Cosmology Astropart. Phys. 8 (Aug., 2016) 069, [1602.07282].

[25] P. D. Serpico, E. Sefusatti, M. Gustafsson and G. Zaharijas, Extragalactic gamma-ray signal from dark matter annihilation: a power spectrum based computation, MNRAS 421 (Mar., 2012) L87-L91, [1109.0095].

[26] E. Sefusatti, G. Zaharijas, P. D. Serpico, D. Theurel and M. Gustafsson, Extragalactic gamma-ray signal from dark matter annihilation: an appraisal, MNRAS 441 (July, 2014) 1861-1878, [1401.2117].

[27] A. Klypin, G. Yepes, S. Gottlöber, F. Prada and S. Heß, MultiDark simulations: the story of dark matter halo concentrations and density profiles, MNRAS 457 (Apr., 2016) 4340-4359, [1411.4001].

[28] A. Rodríguez-Puebla, P. Behroozi, J. Primack, A. Klypin, C. Lee and D. Hellinger, Halo and subhalo demographics with Planck cosmological parameters: Bolshoi-Planck and MultiDark-Planck simulations, MNRAS 462 (Oct., 2016) 893-916, [1602.04813].

[29] C. A. Correa, J. S. B. Wyithe, J. Schaye and A. R. Duffy, The accretion history of dark matter haloes - III. A physical model for the concentration-mass relation, MNRAS 452 (Sept., 2015) 1217-1232, [1502.00391].

[30] A. D. Ludlow, S. Bose, R. E. Angulo, L. Wang, W. A. Hellwing, J. F. Navarro et al., The mass-concentration-redshift relation of cold and warm dark matter haloes, MNRAS 460 (Aug., 2016) 1214-1232, [1601.02624].

[31] Planck Collaboration, P. A. R. Ade, N. Aghanim, M. Arnaud, M. Ashdown, J. Aumont et al., Planck 2015 results. XIII. Cosmological parameters, A\&3A 594 (Sept., 2016) A13, [1502.01589].

[32] J. Tinker, A. V. Kravtsov, A. Klypin, K. Abazajian, M. Warren, G. Yepes et al., Toward a Halo Mass Function for Precision Cosmology: The Limits of Universality, ApJ 688 (Dec., 2008) 709-728, [0803.2706].

[33] M. A. Sánchez-Conde and F. Prada, The flattening of the concentration-mass relation towards low halo masses and its implications for the annihilation signal boost, MNRAS 442 (Aug., 2014) 2271-2277, [1312.1729].

[34] A. Charbonnier, C. Combet and D. Maurin, CLUMPY: A code for $\gamma$-ray signals from dark matter structures, Computer Physics Communications 183 (Mar., 2012) 656-668, [1201.4728]. 
[35] V. Bonnivard, M. Hütten, E. Nezri, A. Charbonnier, C. Combet and D. Maurin, CLUMPY: Jeans analysis, $\gamma$-ray and $\nu$ fluxes from dark matter (sub-)structures, Computer Physics Communications 200 (Mar., 2016) 336-349, [1506.07628].

[36] A. Charbonnier, C. Combet, M. Daniel, S. Funk, J. A. Hinton, D. Maurin et al., Dark matter profiles and annihilation in dwarf spheroidal galaxies: prospectives for present and future $\gamma$-ray observatories - I. The classical dwarf spheroidal galaxies, MNRAS 418 (Dec., 2011) 1526-1556, [1104.0412].

[37] V. Bonnivard, C. Combet, D. Maurin and M. G. Walker, Spherical Jeans analysis for dark matter indirect detection in dwarf spheroidal galaxies - impact of physical parameters and triaxiality, MNRAS 446 (Jan., 2015) 3002-3021, [1407.7822].

[38] V. Bonnivard, C. Combet, M. Daniel, S. Funk, A. Geringer-Sameth, J. A. Hinton et al., Dark matter annihilation and decay in dwarf spheroidal galaxies: the classical and ultrafaint dSphs, MNRAS 453 (Oct., 2015) 849-867, [1504.02048].

[39] C. Combet, D. Maurin, E. Nezri, E. Pointecouteau, J. A. Hinton and R. White, Decaying dark matter: Stacking analysis of galaxy clusters to improve on current limits, Phys. Rev. D $\mathbf{8 5}$ (Mar., 2012) 063517, [1203.1164].

[40] E. Nezri, R. White, C. Combet, J. A. Hinton, D. Maurin and E. Pointecouteau, $\gamma$-rays from annihilating dark matter in galaxy clusters: stacking versus single source analysis, MNRAS 425 (Sept., 2012) 477-489, [1203.1165].

[41] M. Hütten, C. Combet, G. Maier and D. Maurin, Dark matter substructure modelling and sensitivity of the Cherenkov Telescope Array to Galactic dark halos, J. Cosmology Astropart. Phys. 9 (Sept., 2016) 047, [1606. 04898].

[42] E. Charles, M. Sánchez-Conde, B. Anderson, R. Caputo, A. Cuoco, M. Di Mauro et al., Sensitivity projections for dark matter searches with the Fermi large area telescope, Phys. Rep. 636 (June, 2016) 1-46, [1605.02016].

[43] M. Cirelli, G. Corcella, A. Hektor, G. Hütsi, M. Kadastik, P. Panci et al., PPPC 4 DM ID: $a$ poor particle physicist cookbook for dark matter indirect detection, J. Cosmology Astropart. Phys. 3 (Mar., 2011) 051, [1012.4515].

[44] A. V. Belikov and D. Hooper, Contribution of inverse Compton scattering to the diffuse extragalactic gamma-ray background from annihilating dark matter, Phys. Rev. D 81 (Feb., 2010) 043505, [0906.2251].

[45] S. Ando and E. Komatsu, Constraints on the annihilation cross section of dark matter particles from anisotropies in the diffuse gamma-ray background measured with Fermi-LAT, Phys. Rev. D 87 (June, 2013) 123539, [1301.5901].

[46] J. Lesgourgues, The Cosmic Linear Anisotropy Solving System (CLASS) I: Overview, ArXiv e-prints (Apr., 2011), [1104.2932].

[47] D. J. Heath, The growth of density perturbations in zero pressure Friedmann-Lemaitre universes, MNRAS 179 (May, 1977) 351-358.

[48] G. Despali, C. Giocoli, R. E. Angulo, G. Tormen, R. K. Sheth, G. Baso et al., The universality of the virial halo mass function and models for non-universality of other halo definitions, MNRAS 456 (Mar., 2016) 2486-2504, [1507.05627].

[49] A. Domínguez, J. D. Finke, F. Prada, J. R. Primack, F. S. Kitaura, B. Siana et al., Detection of the Cosmic $\gamma$-Ray Horizon from Multiwavelength Observations of Blazars, ApJ 770 (June, 2013) 77, [1305.2162].

[50] M. Kuhlen, M. Vogelsberger and R. Angulo, Numerical simulations of the dark universe: State of the art and the next decade, Physics of the Dark Universe 1 (Nov., 2012) 50-93, [1209.5745]. 
[51] S. Profumo, K. Sigurdson and M. Kamionkowski, What Mass Are the Smallest Protohalos?, Phys. Rev. Lett. 97 (July, 2006) 031301, [astro-ph/0603373].

[52] T. Bringmann, Particle models and the small-scale structure of dark matter, New Journal of Physics 11 (Oct., 2009) 105027, [0903.0189].

[53] V. S. Berezinsky, V. I. Dokuchaev and Y. N. Eroshenko, Small-scale clumps of dark matter, Physics Uspekhi 57 (Jan., 2014) 1-36, [1405.2204].

[54] J. S. Bullock and M. Boylan-Kolchin, Small-Scale Challenges to the $\Lambda$ CDM Paradigm, ARAESA 55 (Aug., 2017) 343-387, [1707.04256].

[55] S. Bocquet, A. Saro, K. Dolag and J. J. Mohr, Halo mass function: baryon impact, fitting formulae, and implications for cluster cosmology, MNRAS 456 (Mar., 2016) 2361-2373, [1502.07357].

[56] E. Komatsu, K. M. Smith, J. Dunkley, C. L. Bennett, B. Gold, G. Hinshaw et al., Seven-year Wilkinson Microwave Anisotropy Probe (WMAP) Observations: Cosmological Interpretation, ApJS 192 (Feb., 2011) 18, [1001.4538].

[57] Y. Inoue, S. Inoue, M. A. R. Kobayashi, R. Makiya, Y. Niino and T. Totani, Extragalactic Background Light from Hierarchical Galaxy Formation: Gamma-Ray Attenuation up to the Epoch of Cosmic Reionization and the First Stars, ApJ 768 (May, 2013) 197, [1212.1683].

[58] A. Franceschini, G. Rodighiero and M. Vaccari, Extragalactic optical-infrared background radiation, its time evolution and the cosmic photon-photon opacity, A $\mathscr{G} A 487$ (Sept., 2008) 837-852, [0805.1841].

[59] A. Domínguez, J. R. Primack, D. J. Rosario, F. Prada, R. C. Gilmore, S. M. Faber et al., Extragalactic background light inferred from AEGIS galaxy-SED-type fractions, MNRAS $\mathbf{4 1 0}$ (Feb., 2011) 2556-2578, [1007.1459].

[60] R. C. Gilmore, R. S. Somerville, J. R. Primack and A. Domínguez, Semi-analytic modelling of the extragalactic background light and consequences for extragalactic gamma-ray spectra, MNRAS 422 (June, 2012) 3189-3207, [1104.0671].

[61] T. Ishiyama, Hierarchical Formation of Dark Matter Halos and the Free Streaming Scale, ApJ 788 (June, 2014) 27, [1404.1650].

[62] V. Springel, J. Wang, M. Vogelsberger, A. Ludlow, A. Jenkins, A. Helmi et al., The Aquarius Project: the subhaloes of galactic haloes, MNRAS 391 (Dec., 2008) 1685-1711, [0809.0898].

[63] L. Gao, J. F. Navarro, C. S. Frenk, A. Jenkins, V. Springel and S. D. M. White, The Phoenix Project: the dark side of rich Galaxy clusters, MNRAS 425 (Sept., 2012) 2169-2186, [1201.1940].

[64] C. Patrignani and P. D. Group, Review of particle physics, Chinese Physics C 40 (2016) 100001

[65] G. L. Bryan and M. L. Norman, Statistical Properties of X-Ray Clusters: Analytic and Numerical Comparisons, ApJ 495 (Mar., 1998) 80-99, [astro-ph/9710107].

[66] M. Bonamigo, G. Despali, M. Limousin, R. Angulo, C. Giocoli and G. Soucail, Universality of dark matter haloes shape over six decades in mass: insights from the Millennium XXL and SBARBINE simulations, MNRAS 449 (May, 2015) 3171-3182, [1410.0015].

[67] G. Despali, C. Giocoli, M. Bonamigo, M. Limousin and G. Tormen, A look into the inside of haloes: a characterization of the halo shape as a function of overdensity in the Planck cosmology, MNRAS 466 (Apr., 2017) 181-193, [1605.04319].

[68] J. F. Navarro, C. S. Frenk and S. D. M. White, The Structure of Cold Dark Matter Halos, ApJ 462 (May, 1996) 563, [astro-ph/9508025]. 
[69] J. Einasto and U. Haud, Galactic models with massive corona. I - Method. II - Galaxy, A\&A 223 (Oct., 1989) 89-106.

[70] J. Stadel, D. Potter, B. Moore, J. Diemand, P. Madau, M. Zemp et al., Quantifying the heart of darkness with GHALO - a multibillion particle simulation of a galactic halo, MNRAS $\mathbf{3 9 8}$ (Sept., 2009) L21-L25, [0808. 2981].

[71] A. A. Dutton and A. V. Macciò, Cold dark matter haloes in the Planck era: evolution of structural parameters for Einasto and NFW profiles, MNRAS 441 (July, 2014) 3359-3374, [1402.7073].

[72] M. A. Henson, D. J. Barnes, S. T. Kay, I. G. McCarthy and J. Schaye, The impact of baryons on massive galaxy clusters: halo structure and cluster mass estimates, MNRAS 465 (Mar., 2017) 3361-3378, [1607.08550].

[73] B. F. Griffen, A. P. Ji, G. A. Dooley, F. A. Gómez, M. Vogelsberger, B. W. O’Shea et al., The Caterpillar Project: A Large Suite of Milky Way Sized Halos, ApJ 818 (Feb., 2016) 10, [1509.01255].

[74] L. Gao, J. F. Navarro, S. Cole, C. S. Frenk, S. D. M. White, V. Springel et al., The redshift dependence of the structure of massive $\Lambda$ cold dark matter haloes, MNRAS $\mathbf{3 8 7}$ (June, 2008) 536-544, [0711.0746].

[75] A. D. Ludlow and R. E. Angulo, Einasto profiles and the dark matter power spectrum, MNRAS 465 (Feb., 2017) L84-L88, [1610.04620].

[76] S. Peirani, Y. Dubois, M. Volonteri, J. Devriendt, K. Bundy, J. Silk et al., Density profile of dark matter haloes and galaxies in the horizon-agn simulation: the impact of AGN feedback, MNRAS 472 (Dec., 2017) 2153-2169, [1611.09922].

[77] K. Umetsu and B. Diemer, Lensing Constraints on the Mass Profile Shape and the Splashback Radius of Galaxy Clusters, ApJ 836 (Feb., 2017) 231, [1611.09366].

[78] O. Host and S. H. Hansen, A Detailed Statistical Analysis of the Mass Profiles of Galaxy Clusters, ApJ 736 (July, 2011) 52, [0907.1097].

[79] A. B. Mantz, S. W. Allen and R. G. Morris, Cosmology and astrophysics from relaxed galaxy clusters - V. Consistency with cold dark matter structure formation, MNRAS 462 (Oct., 2016) 681-688, [1607.04686].

[80] J. S. Bullock, T. S. Kolatt, Y. Sigad, R. S. Somerville, A. V. Kravtsov, A. A. Klypin et al., Profiles of dark haloes: evolution, scatter and environment, MNRAS 321 (Mar., 2001) 559-575, [astro-ph/9908159].

[81] V. R. Eke, J. F. Navarro and M. Steinmetz, The Power Spectrum Dependence of Dark Matter Halo Concentrations, ApJ 554 (June, 2001) 114-125, [astro-ph/0012337].

[82] D. H. Zhao, Y. P. Jing, H. J. Mo and G. Börner, Mass and Redshift Dependence of Dark Halo Structure, ApJ 597 (Nov., 2003) L9-L12, [astro-ph/0309375].

[83] J. Merten, M. Meneghetti, M. Postman, K. Umetsu, A. Zitrin, E. Medezinski et al., CLASH: The Concentration-Mass Relation of Galaxy Clusters, ApJ 806 (June, 2015) 4, [1404.1376].

[84] S. Amodeo, S. Ettori, R. Capasso and M. Sereno, The relation between mass and concentration in X-ray galaxy clusters at high redshift, AEA 590 (May, 2016) A126, [1604.02163].

[85] A. Biviano, A. Moretti, A. Paccagnella, B. M. Poggianti, D. Bettoni, M. Gullieuszik et al., The concentration-mass relation of clusters of galaxies from the OmegaWINGS survey, ArXiv e-prints (Aug., 2017), [1708.07349].

[86] K. Dolag, M. Bartelmann, F. Perrotta, C. Baccigalupi, L. Moscardini, M. Meneghetti et al., Numerical study of halo concentrations in dark-energy cosmologies, A\& A 416 (Mar., 2004) 853-864, [astro-ph/0309771]. 
[87] A. Cooray, Extragalactic background light measurements and applications, Royal Society Open Science 3 (Mar., 2016) 150555, [1602.03512].

[88] M. G. Hauser and E. Dwek, The Cosmic Infrared Background: Measurements and Implications, ARAËA 39 (2001) 249-307, [astro-ph/0105539].

[89] J. Binney and S. Tremaine, Galactic Dynamics: Second Edition. Princeton University Press, 2008.

[90] S. Hofmann, D. J. Schwarz and H. Stöcker, Damping scales of neutralino cold dark matter, Phys. Rev. D 64 (Oct., 2001) 083507, [astro-ph/0104173].

[91] V. Berezinsky, V. Dokuchaev and Y. Eroshenko, Small-scale clumps in the galactic halo and dark matter annihilation, Phys. Rev. D 68 (Nov., 2003) 103003, [astro-ph/0301551].

[92] A. M. Green, S. Hofmann and D. J. Schwarz, The power spectrum of SUSY-CDM on subgalactic scales, MNRAS 353 (Sept., 2004) L23-L27, [astro-ph/0309621].

[93] A. M. Green, S. Hofmann and D. J. Schwarz, The first WIMPy halos, J. Cosmology Astropart. Phys. 8 (Aug., 2005) 003, [astro-ph/0503387].

[94] T. Bringmann and S. Hofmann, Thermal decoupling of WIMPs from first principles, J. Cosmology Astropart. Phys. 4 (Apr., 2007) 016, [hep-ph/0612238].

[95] A. Loeb and M. Zaldarriaga, Small-scale power spectrum of cold dark matter, Phys. Rev. D 71 (May, 2005) 103520, [astro-ph/0504112].

[96] E. Bertschinger, Effects of cold dark matter decoupling and pair annihilation on cosmological perturbations, Phys. Rev. D 74 (Sept., 2006) 063509, [astro-ph/0607319].

[97] J. Diemand, B. Moore and J. Stadel, Earth-mass dark-matter haloes as the first structures in the early Universe, Nature 433 (Jan., 2005) 389-391, [astro-ph/0501589].

[98] T. Ishiyama, J. Makino and T. Ebisuzaki, Gamma-ray Signal from Earth-mass Dark Matter Microhalos, ApJ 723 (Nov., 2010) L195-L200, [1006.3392].

[99] D. Anderhalden and J. Diemand, Density profiles of CDM microhalos and their implications for annihilation boost factors, J. Cosmology Astropart. Phys. 4 (Apr., 2013) 009, [1302.0003].

[100] G. Ogiya, D. Nagai and T. Ishiyama, Dynamical evolution of primordial dark matter haloes through mergers, MNRAS 461 (Sept., 2016) 3385-3396, [1604.02866].

[101] R. E. Angulo, O. Hahn, A. D. Ludlow and S. Bonoli, Earth-mass haloes and the emergence of NFW density profiles, MNRAS 471 (Nov., 2017) 4687-4701, [1604.03131].

[102] G. Ogiya and O. Hahn, What sets the central structure of dark matter haloes?, ArXiv e-prints (July, 2017) , [1707.07693].

[103] J. Lavalle, Q. Yuan, D. Maurin and X.-J. Bi, Full calculation of clumpiness boost factors for antimatter cosmic rays in the light of $\Lambda C D M N$-body simulation results. Abandoning hope in clumpiness enhancement?, A $\mathscr{S} A 479$ (Feb., 2008) 427-452, [0709.3634].

[104] J. Diemand, M. Kuhlen and P. Madau, Dark Matter Substructure and Gamma-Ray Annihilation in the Milky Way Halo, ApJ 657 (Mar., 2007) 262-270, [astro-ph/0611370].

[105] W. A. Hellwing, C. S. Frenk, M. Cautun, S. Bose, J. Helly, A. Jenkins et al., The Copernicus Complexio: a high-resolution view of the small-scale Universe, MNRAS 457 (Apr., 2016) 3492-3509, [1505.06436].

[106] S. Garrison-Kimmel, A. Wetzel, J. S. Bullock, P. F. Hopkins, M. Boylan-Kolchin, C.-A. Faucher-Giguère et al., Not so lumpy after all: modelling the depletion of dark matter subhaloes by Milky Way-like galaxies, MNRAS 471 (Oct., 2017) 1709-1727, [1701.03792].

[107] J. Comparat, F. Prada, G. Yepes and A. Klypin, Accurate mass and velocity functions of dark matter haloes, MNRAS 469 (Aug., 2017) 4157-4174, [1702.01628]. 
[108] F. Jiang and F. C. van den Bosch, Statistics of dark matter substructure - III. Halo-to-halo variance, MNRAS 472 (Nov., 2017) 657-674, [1610.02399].

[109] F. C. van den Bosch and F. Jiang, Statistics of dark matter substructure - II. Comparison of model with simulation results, MNRAS 458 (May, 2016) 2870-2884.

[110] P. Mollitor, E. Nezri and R. Teyssier, Baryonic and dark matter distribution in cosmological simulations of spiral galaxies, MNRAS 447 (Feb., 2015) 1353-1369, [1405.4318].

[111] M. Stref and J. Lavalle, Modeling dark matter subhalos in a constrained galaxy: Global mass and boosted annihilation profiles, Phys. Rev. D 95 (Mar., 2017) 063003, [1610.02233].

[112] R. Bartels and S. Ando, Boosting the annihilation boost: Tidal effects on dark matter subhalos and consistent luminosity modeling, Phys. Rev. D 92 (Dec., 2015) 123508, [1507.08656].

[113] Á. Moliné, M. A. Sánchez-Conde, S. Palomares-Ruiz and F. Prada, Characterization of subhalo structural properties and implications for dark matter annihilation signals, MNRAS 466 (Apr., 2017) 4974-4990, [1603.04057].

[114] M. Ackermann, M. Ajello, A. Albert, W. B. Atwood, L. Baldini, J. Ballet et al., The Spectrum of Isotropic Diffuse Gamma-Ray Emission between $100 \mathrm{MeV}$ and 820 GeV, ApJ 799 (Jan., 2015) 86, [1410.3696].

[115] B. Moore, Evidence against dissipation-less dark matter from observations of galaxy haloes, Nature 370 (Aug., 1994) 629-631.

[116] M. Kaplinghat, S. Tulin and H.-B. Yu, Dark Matter Halos as Particle Colliders: Unified Solution to Small-Scale Structure Puzzles from Dwarfs to Clusters, Physical Review Letters 116 (Jan., 2016) 041302, [1508.03339].

[117] K. A. Oman, J. F. Navarro, A. Fattahi, C. S. Frenk, T. Sawala, S. D. M. White et al., The unexpected diversity of dwarf galaxy rotation curves, MNRAS 452 (Oct., 2015) 3650-3665, [1504.01437].

[118] J. S. Bullock, Notes on the Missing Satellites Problem, ArXiv e-prints (Sept., 2010), [1009.4505].

[119] M. Boylan-Kolchin, J. S. Bullock and M. Kaplinghat, Too big to fail? The puzzling darkness of massive Milky Way subhaloes, MNRAS 415 (July, 2011) L40-L44, [1103.0007].

[120] S. Tulin and H.-B. Yu, Dark Matter Self-interactions and Small Scale Structure, ArXiv e-prints (May, 2017) , [1705.02358].

[121] S. W. Randall, M. Markevitch, D. Clowe, A. H. Gonzalez and M. Bradač, Constraints on the Self-Interaction Cross Section of Dark Matter from Numerical Simulations of the Merging Galaxy Cluster 1E 0657-56, ApJ 679 (June, 2008) 1173-1180, [0704.0261].

[122] A. H. G. Peter, M. Rocha, J. S. Bullock and M. Kaplinghat, Cosmological simulations with self-interacting dark matter - II. Halo shapes versus observations, MNRAS 430 (Mar., 2013) 105-120, [1208.3026].

[123] J. Zavala, M. Vogelsberger and M. G. Walker, Constraining self-interacting dark matter with the Milky Way's dwarf spheroidals, MNRAS 431 (Apr., 2013) L20-L24, [1211.6426].

[124] C. Bœehm, J. A. Schewtschenko, R. J. Wilkinson, C. M. Baugh and S. Pascoli, Using the Milky Way satellites to study interactions between cold dark matter and radiation, MNRAS 445 (Nov., 2014) L31-L35, [1404.7012].

[125] J. A. Schewtschenko, R. J. Wilkinson, C. M. Baugh, C. Bohm and S. Pascoli, Dark matter-radiation interactions: the impact on dark matter haloes, MNRAS 449 (June, 2015) 3587-3596, [1412.4905]. 
[126] M. Rocha, A. H. G. Peter, J. S. Bullock, M. Kaplinghat, S. Garrison-Kimmel, J. Oñorbe et al., Cosmological simulations with self-interacting dark matter - I. Constant-density cores and substructure, MNRAS 430 (Mar., 2013) 81-104, [1208.3025].

[127] A. Burkert, The Structure of Dark Matter Halos in Dwarf Galaxies, ApJ 447 (July, 1995) L25-L28, [astro-ph/9504041].

[128] C. Giocoli, G. Tormen, R. K. Sheth and F. C. van den Bosch, The substructure hierarchy in dark matter haloes, MNRAS 404 (May, 2010) 502-517, [0911.0436]. 